\title{
Antibody-mediated targeting of iron oxide nanoparticles to the folate receptor alpha increases tumor cell association in vitro and in vivo
}

This article was published in the following Dove Press journal:

International Journal of Nanomedicine

I April 2015

Number of times this article has been viewed

Christian Ndong'

Seiko Toraya-Brown ${ }^{2}$

Katsiaryna Kekalo'

Ian Baker

Tillman U Gerngross ${ }^{1,3,4}$

Steven N Fiering $2,5,6$

Karl E Griswold $1,3,6$

'Thayer School of Engineering, Dartmouth, Hanover, NH, USA;

2Department of Microbiology and Immunology, Geisel School of Medicine at Dartmouth, Hanover, $\mathrm{NH}$, USA; ${ }^{3}$ Department of Biological Sciences, Dartmouth, Hanover, NH, USA; ${ }^{4}$ Department of Chemistry,

Dartmouth, Hanover, NH, USA;

${ }^{5}$ Department of Genetics, Geisel School of Medicine at Dartmouth, Hanover, NH, USA; ${ }^{\circ}$ Norris Cotton Cancer Center, Lebanon, NH, USA

Correspondence: Karl E Griswold Thayer School of Engineering, Dartmouth, Hanover, NH 03755, USA

Tel + I 6036462127

Fax +I 6036462277

Email karl.e.griswold@dartmouth.edu
Abstract: Active molecular targeting has become an important aspect of nanoparticle development for oncology indications. Here, we describe molecular targeting of iron oxide nanoparticles (IONPs) to the folate receptor alpha (FOLR $\alpha)$ using an engineered antibody fragment (Ffab). Compared to control nanoparticles targeting the non-relevant botulinum toxin, the Ffab-IONP constructs selectively accumulated on FOLR $\alpha$-overexpressing cancer cells in vitro, where they exhibited the capacity to internalize into intracellular vesicles. Similarly, Ffab-IONPs homed to FOLR $\alpha$-positive tumors upon intraperitoneal administration in an orthotopic murine xenograft model of ovarian cancer, whereas negative control particles showed no detectable tumor accumulation. Interestingly, Ffab-IONPs built with custom $120 \mathrm{~nm}$ nanoparticles exhibited lower in vitro targeting efficiency when compared to those built with commercially sourced $180 \mathrm{~nm}$ nanoparticles. In vivo, however, the two Ffab-IONP platforms achieved equivalent tumor homing, although the smaller $120 \mathrm{~nm}$ IONPs were more prone to liver sequestration. Overall, the results show that Ffab-mediated targeting of IONPs yields specific, high-level accumulation within cancer cells, and this fact suggests that Ffab-IONPs could have future utility in ovarian cancer diagnostics and therapy.

Keywords: nanoparticle targeting, antibody fragment, biodistribution, ovarian cancer

\section{Introduction}

Despite widespread advances in cancer diagnostics and treatment, ovarian cancers continue to have high mortality, with 5 -year survival rates remaining near $45 \%$ since the mid-1990s. ${ }^{1}$ Hyperthermia represents one promising approach for peritoneal cancer therapy, as this modality has the capacity to kill cancer cells in a direct fashion and also indirectly stimulates an anticancer immune response..$^{2-6}$ In seeking to apply hyperthermia therapy to dispersed peritoneal tumors, however, delivering thermal doses to malignant cells in a precise and controlled fashion represents a substantial technical barrier.

Iron oxide nanoparticles (IONPs) have a decades-long history as heating mediators in hyperthermia, ${ }^{7}$ and advances in nanoparticle fabrication and functionalization have fueled further interest in this research space. ${ }^{8-10}$ Preferential accumulation of IONPs in the tumor, however, remains a challenge in balancing efficacy and safety. ${ }^{8,11}$ One recent study co-opted tumor-associated peritoneal phagocytes to selectively deliver IONPs in an ovarian cancer model. ${ }^{6}$ In other work, luteinizing hormone-releasing hormone (LHRH) peptide was used as an IONP-targeting moiety for ovarian cancer cells overexpressing the LHRH receptor. ${ }^{12}$ Similarly, many ovarian cancers overexpress folate receptor alpha (FOLR $\alpha){ }^{13,14}$ and this fact has been leveraged to selectively 
target IONPs via functionalization with the cognate folic acid ligand. ${ }^{15}$ Monoclonal antibodies and antibody fragments have also been used to selectively target IONPs to ovarian cancer cells, ${ }^{16,17}$ but to date there is no report of antibody-mediated IONP targeting to the FOLR $\alpha$ surface protein. Antibody targeting of FOLR $\alpha$ might offer performance advantages over targeting with the folic acid ligand, as the former should be highly specific to FOLR $\alpha$, while the latter is also bound with high affinity by folate receptors beta and gamma, and can interfere with uptake of circulating folate in patients. ${ }^{18,19}$

In the current research, we describe the development and characterization of IONPs functionalized with an engineered fab fragment of Farletuzumab, a humanized monoclonal antibody that has demonstrated tumor-inhibitory effects in pre-clinical models ${ }^{20-22}$ and in clinical trials. ${ }^{23}$ Tumor-specific homing of the antibody fragment Farletuzufab (Ffab)-targeted IONPs was assessed both in vitro and in vivo, and the results were compared to negative control particles targeting an irrelevant protein. In aggregate, these studies demonstrate the performance advantage of IONPs that actively target the FOLR $\alpha$ cancer marker.

\section{Materials and methods}

\section{Cells lines and culture conditions}

$\mathrm{KB}$ cells, derived from a human squamous cell carcinoma of the oral cavity, were obtained as a gift from Dr Philip S Low at Purdue University (West Lafayette, IN, USA). These $\mathrm{KB}$ cells were found to produce disseminated peritoneal tumors that are representative of advanced ovarian cancer in humans. The cells were maintained as a monolayer in folate-free Roswell Park Memorial Institute (RPMI) 1640 medium (Thermo Fisher Scientific, Waltham, MA, USA) supplemented with $100 \mathrm{U} / \mathrm{mL}$ penicillin, $100 \mu \mathrm{g} / \mathrm{mL}$ streptomycin, and $10 \%$ fetal bovine serum (FBS) at $37^{\circ} \mathrm{C}$ in a humidified atmosphere consisting of $5 \% \mathrm{CO}_{2}$ and $95 \%$ air. Cells were harvested with $0.25 \%$ trypsin, suspended, and spun down at 1,200 rpm prior to re-suspension and use in subsequent experiments.

\section{Construction of Ffab and anti-botulinum toxin fab fragments}

Ffab and negative control anti-botulinum toxin fab fragment (Bfab) were reformatted from their corresponding full length immunoglobulin G (IgG) monoclonal antibody sequences, which are available from the literature. ${ }^{24,25}$ Coding sequences for the variable and constant regions of the heavy and light chains from respective full length IgGs were reverse translated, codon optimized for expression in mammalian cells, and synthesized by DNA 2.0 (DNA 2.0 Inc., Menlo Park, CA, USA). Both Ffab and Bfab heavy chains were designed to carry an engineered cysteine tag at their respective C-termini for subsequent site-specific conjugation to maleimide-polyethylene glycol 2 (PEG2)-biotin (Thermo Fisher Scientific) or nanoparticles. Ffab and Bfab light- and heavy-chain constructs were individually sub-cloned into the CMVR VRC01 expression vector (National Institutes of Health [NIH] acquired immunodeficiency syndrome [AIDS] Reagent Program, Germantown, MD, USA).

\section{Expression, extraction, and purification of Ffab protein}

CMVR VRC01 expression vectors separately harboring Ffab light chain and heavy chain or Bfab light chain and heavy chain were co-transfected into suspension HEK 293 cells using polyethylenimine (Polysciences Inc, Warrington, PA, USA) as previously described. ${ }^{26}$ Secreted Ffab and Bfab were clarified through centrifugation at $8,000 \mathrm{rpm}$ at $4^{\circ} \mathrm{C}$ for 15 minutes on an Avanti ${ }^{\circledR}$ J 25 centrifuge (Beckman Coulter Inc, Brea, CA, USA). The resulting supernatants were filtered through a $0.45 \mu \mathrm{m}$ filter to remove any residual cell debris and other large particles before loading onto a fast protein liquid chromatography (FPLC) affinity column for purification.

Affinity purification was performed on a pre-packed 5 mL KappaSelect column from GE Healthcare Bio-Sciences Corporation (Piscataway, NJ, USA) as suggested by manufacturer instructions. Fabs were eluted with $100 \mathrm{mM}$ glycine at $\mathrm{pH} 2.7$ in $2 \mathrm{~mL}$ Eppendorf tubes prefilled with $50 \mu \mathrm{L}$ of $1 \mathrm{M}$ tris(hydroxymethyl)aminomethane (Tris), $5 \mathrm{mM}$ ethylenediaminetetraacetic acid (EDTA). The purification process was automated on an AKTA ${ }^{\text {TM }}$ FPLC system (GE Healthcare Bio-Sciences Corp). Purified proteins were subjected to a second step size exclusion chromatography column using Superdex $^{\circledR} 75$ (GE Healthcare Bio-Sciences Corp). Final products were eluted in phosphate-buffered saline (PBS) and stored at $-20^{\circ} \mathrm{C}$ until used.

Reductive activation and chemical conjugation of the purified fabs to maleimide-PEG2-biotin (Thermo Fisher Scientific) were performed as described previously. ${ }^{26}$ Briefly, maleimide-PEG2-biotin was added to a final concentration of $2 \mathrm{mM}$ (10 to 1 molar excess for maleimide-PEG2-biotin to proteins). The conjugation reaction was allowed to proceed for 2 hours at room temperature. The excess of maleimidePEG2-biotin was removed by buffer exchange through a HiTrap ${ }^{\mathrm{TM}}$ desalting column using PBS pH 7.0, and the resultant Bfab and Ffab conjugates (Bfab-maleimide-PEG2-biotin 
and Ffab-maleimide-PEG2-biotin) were stored at $-20^{\circ} \mathrm{C}$ until further use. Purified and conjugated proteins were analyzed using non-reduced and reduced sodium dodecyl sulfate polyacrylamide gel electrophoresis (SDS-PAGE) conditions and stained with Coomassie blue. Protein constructs were verified further using electrospray ionization time-of-flight (ESITOF) liquid chromatography mass spectrometry (Agilent Technologies, Santa Clara, CA, USA).

\section{Affinity measurement of Ffab}

The affinity of Ffab protein was analyzed using enzymelinked immunosorbent assay (ELISA) and biolayer interferometry on the ForteBio Octet ${ }^{\circledR}$ Red instrument (ForteBio, Menlo Park, CA, USA). For ELISA, commercial rFOLR1-his protein (Sino Biological Inc, Beijing, People's Republic of China) reconstituted at $50 \mu \mathrm{g} / \mathrm{mL}(1.9 \mu \mathrm{M})$ in water was diluted in coating buffer $(100 \mathrm{mM}$ sodium carbonate at $\mathrm{pH}$ 9.4) to $100 \mathrm{ng} / \mathrm{mL}$ (3 nM). Two 96-well Immulon ${ }^{\mathrm{TM}} 4 \mathrm{HBX}$ high protein binding plates (Thermo Fisher Scientific) were coated with $100 \mu \mathrm{L}$ of rFOLR1-his at $4^{\circ} \mathrm{C}$ for 16 hours. Bindings with serial dilutions of Ffab and Bfab (0-200 nM) were performed as previously described. ${ }^{26}$ The monovalent affinity of Ffab was measured by biolayer interferometry. Briefly, rFOLR1-his was loaded in a 96-well plate at different concentrations (1.56-25 nM). Ffab was coupled to streptavidin biosensor tips (ForteBio) at $20 \mu \mathrm{g} / \mathrm{mL}$ and immersed into the rFOLR1-his dilutions. Ffab association and dissociation rates and equilibrium affinity were determined using software provided with the instrument.

\section{Ffab cell-binding studies}

For live cell binding, KB cells were harvested from T250 flasks (USA Scientific, Ocala, FL, USA) and seeded into a 96-well microplate at 50,000 cells per well using PBS containing $2 \%$ FBS. Different concentrations of Ffab or Bfab (0-200 nM) were added to cells and incubated at room temperature on a shaker (150 rpm) for 1 hour. Plates were centrifuged at 1,200 rpm for 5 minutes and washed twice with cold PBS containing $2 \%$ FBS using a vacuum unit. Cells were incubated with Phycolink ${ }^{\circledR}$ Streptavidin-R-Phycoerythrin (Prozyme, Hayward, CA, USA) at room temperature for 30 minutes, centrifuged, and washed as in previous step. Cells were resuspended in PBS, $2 \%$ FBS, and analyzed on a MacsQuant ${ }^{\mathrm{B}}$ instrument (Miltenyi Biotec Inc, Auburn, CA, USA).

\section{Production of carboxymethyl dextran IONPs}

Commercially available ferric chloride $\left(\mathrm{FeCl}_{3} \cdot 6 \mathrm{H}_{2} \mathrm{O}\right)$, ferrous sulfate $\left(\mathrm{FeSO}_{4} \cdot 7 \mathrm{H}_{2} \mathrm{O}\right), 25 \mathrm{wt} \%$ ammonium hydroxide solution, $\mathrm{NaNO}_{3}$, and $\mathrm{NaOH}$ were purchased from VWR International (Radnor, PA, USA). Carboxymethyl-dextran (CMD) $40 \mathrm{kDa}$ was purchased from TdB Consultancy AB (Uppsala, Sweden). All reactants were used as received without further purification. Magnetic nanoparticles (MNPs) with CMD embedded in their structure, as described by Kekalo and Baker, ${ }^{27}$ were also obtained. Briefly, $10 \%$ solutions of salts of $\mathrm{Fe}$ and $\mathrm{Fe}$ (III) were precipitated by ammonia solution in the presence of excess of polysaccharide. The mixture was placed on a sand bath and heated up to $70^{\circ} \mathrm{C}$. Then, $\mathrm{NaOH}$ and $\mathrm{NaNO}_{3}$ were added to oxidize Fe and maintain alkali media $(\mathrm{pH}>10)$. The temperature was raised up to $100^{\circ} \mathrm{C}$ at a speed of $10^{\circ} \mathrm{C} /$ hour. The resulting solution was spun at $5,000 \mathrm{rpm}$ for 15 minutes to remove large aggregates. The remaining MNPs were purified using an LS magnetic column separator (Miltenyi Biotec).

\section{Nanoparticle characterization}

Transmission electron micrographs of the nanoparticles were taken using an FEI Technai F20ST field emission gun transmission electron microscope operated at $200 \mathrm{kV}$. Five hundred MNPs from three different locations on a grid were used to produce frequency vs particle size histograms.

\section{Antibody-IONP conjugation and characterization}

The $25 \mathrm{~nm}$ core size CMD-coated nanoparticles were obtained from the Dartmouth nanoparticle core facility, and the $100 \mathrm{~nm}$ core size aminodextran-coated bionized nanoferrite (BNF) nanoparticles were purchased from Micromod Partikeltechnologie GmbH (Rostock, Germany). Prior to use, CMD and BNF-IONPs were purified using MACS ${ }^{\circledR}$ separation LS columns (Miltenyi Biotec) and eluted with sterile water. CMD nanoparticles were maleimide functionalized by adding N-(2-Aminoethyl)maleimide and 1-ethyl-3-(3dimethylaminopropyl)carboiimide (EDC) (both purchased from Sigma-Aldrich Co, St Louis, MO, USA) in 100-fold molar excess and incubating for 2 hours at room temperature in $100 \mathrm{mM}$ 2-morpholinoethanesulfonic acid (MES) $\mathrm{pH}$ 6.3. BNF nanoparticles were maleimide functionalized by adding sulfo-GMBS ( $\mathrm{N}$-[ $\gamma$-maleimobutyryloxy] sulfosuccinimide ester) (Thermo Fisher Scientific) in 100-fold molar excess and incubating at room temperature for 2 hours in $100 \mathrm{mM}$ sodium phosphate buffer $\mathrm{pH}$ 7.2. The excess $\mathrm{N}$-(2-Aminoethyl)maleimide, EDC, and sulfo-GMBS were removed by buffer exchange with $30 \mathrm{mM}$ MES pH 6.5 (for CMD particles) or $30 \mathrm{mM}$ MES, $5 \mathrm{mM}$ EDTA pH 6.5 (for BNF particles) using MACS separation LS columns. 
Purified Ffab and Bfab were reduced with $20 \mathrm{mM}$ cysteine as described previously, ${ }^{26}$ followed by buffer exchange on HiTrap desalting columns (GE Healthcare Bio-Sciences Corp) with $30 \mathrm{mM}$ MES pH 6.5 (for CMD particles) or $30 \mathrm{mM}$ MES, $5 \mathrm{mM}$ EDTA pH 6.5 (for BNF particles). CMD-cysteine-reduced Ffab and Bfab were added to the maleimide-functionalized CMD and BNF-IONPs at a 1:10 $(\mathrm{w} / \mathrm{w})$ ratio and incubated at room temperature for 16 hours at $4^{\circ} \mathrm{C}$ on a shaker set at $125 \mathrm{rpm}$. This ratio was empirically determined to yield high-binding IONP conjugates and reproducible conjugation results. The unbound protein was then removed using MACS separation LS columns and magnetic field. All processes were performed in a sterile environment using sterile and endotoxin-free buffers. Micro BCA assay (Thermo Fisher Scientific) was used to determine the amount of Ffab and Bfab covalently bound to IONPs, as described previously. ${ }^{26}$

The hydrodynamic Z-average diameters (HDD) and zeta potentials $(\mathrm{mV})$ of IONP-Fab conjugates were measured using a Zetasizer Nano ZS (Malvern Instruments, Malvern, UK). For measuring the HDD, the IONPs were diluted to $0.05 \mathrm{mg} / \mathrm{mL}$ in PBS. For the determination of zeta potential, the IONPs were diluted to a concentration of $0.2 \mathrm{mg} / \mathrm{mL}$ in $10 \mathrm{mM} \mathrm{NaCl}$.

\section{In vitro IONP-fab binding studies}

The commercial rFOLR1-his (Sino Biological Inc) was diluted in PBS to $500 \mathrm{ng} / \mathrm{mL}$ ( $20 \mathrm{nM}$ ), and a 96-well Immulon 4HBX high-protein binding plate (Thermo Fisher Scientific) was coated with $100 \mu \mathrm{L}$ of rFOLR-his at $4^{\circ} \mathrm{C}$ for 16 hours. Coating buffer was removed by aspiration and replaced with $300 \mu \mathrm{L}$ of blocking buffer ( $2 \%$ bovine serum albumin [BSA; $\mathrm{w} / \mathrm{v}]$ in $1 \times \mathrm{PBS}, \mathrm{pH} 7.4)$. Serial dilutions of Ffab-CMD and Bfab-CMD (0-5 nM) or Ffab-BNF and Bfab-BNF (0-1 nM) in sample diluents $(0.1 \%$ BSA [w/v] in PBS, pH 7.4) were added and incubated at room temperature for 1 hour. Nanoparticle uptake was measured using a ferrozine-based iron assay as described previously. ${ }^{26}$ Briefly, plates were washed three times using washing buffer (Tris buffer saline [TBS], $0.05 \%$ Tween $20[\mathrm{v} / \mathrm{v}])$. After washing, $100 \mu \mathrm{L}$ of $1.4 \mathrm{M} \mathrm{HCl}$ was added to each well, and plates were sealed and heated at $70^{\circ} \mathrm{C}$ for 2 hours. Plates were centrifuged at $200 \mathrm{rpm}$ to settle liquids, $100 \mu \mathrm{L}$ of ferrozine reagent $(6.5 \mathrm{mM}$ ferrozine, $13 \mathrm{mM}$ neocuproine, and $2 \mathrm{M}$ ascorbic acid diluted in $5 \mathrm{M}$ ammonium acetate) were added to each well, and plates were shaken for 5 minutes. Plates were read at $562 \mathrm{~nm}$ (using a SpectraMax ${ }^{\circledR}$ 190; Molecular Devices LLC, Sunnyvale, CA, USA), and the amount of iron per well was determined from a standard curve of iron $\left(\mathrm{FeCl}_{3}\right)$ in identically processed samples.

For cellular binding, KB cells were seeded at 100,000 cells per well on a 48-well plate (Corning Incorporated, Corning, NY, USA) and allowed to attach overnight. Old medium was removed and replaced with $300 \mu \mathrm{L}$ of fresh medium containing $35 \mu \mathrm{g} / \mathrm{mL}$ of CMD-Ffab (0.6 nM), CMD-Bfab (0.6 nM), BNF-Ffab (0.035 nM), or BNF-Bfab (0.035 nM). Cells were incubated at $37^{\circ} \mathrm{C}$ for 8 hours, and unbound nanoparticles were removed by washing three times with PBS. Cells were treated with $100 \mu \mathrm{L}$ of sodium hydroxide $(\mathrm{NaOH})$, placed on a shaker in a $37^{\circ} \mathrm{C}$ room for 1 hour, and the ferrozine assay was performed as described above in this section, except $300 \mu \mathrm{L}$ of ferrozine reagent was added. ${ }^{26}$

For transmission electron microscopy, KB cells treated with IONPs for 8 hours were washed with PBS and fixed with a fixative solution (3\% glutaraldehyde and $1 \%$ paraformaldehyde in $0.1 \mathrm{M}$ sodium cacodylate, $\mathrm{pH} 7.4$ ) at room temperature for 15 minutes. After incubation, cells were briefly centrifuged and fresh fixative solution was added, followed by an additional 16 hours incubation at $4^{\circ} \mathrm{C}$. Fixed cells were submitted to the Dartmouth Medical Electron Microscope Facility for imaging.

\section{Peritoneal tumor model and IONP biodistribution}

All mice were cared for according to approved Institutional Animal Care and Use Committee (IACUC) animal protocol. NOD.Cg-Prkde ${ }^{\text {scid }} \mathrm{I} 12 \mathrm{rg}^{\mathrm{tm} l \mathrm{Wj} /} / \mathrm{SzJ}$ (NSG) mice were originally obtained from Jackson ImmunoResearch Laboratories, Inc., West Grove, PA, USA, and were bred at the Geisel School of Medicine at Dartmouth. At 5-6 weeks old, female mice were injected intraperitoneally (IP) on day 0 with $2 \times 10^{6} \mathrm{~KB}$ cells in $400 \mu \mathrm{L}$ PBS. Within 2 weeks, tumors started growing on the peritoneal wall and in the peritoneal cavity, much like human ovarian cancer. For testing IONP biodistribution, Ffab-CMD, Bfab-CMD, Ffab-BNF, or Bfab-BNF were injected IP at $750 \mu \mathrm{g}$ iron in $400 \mu \mathrm{L}$ PBS on day 18 . Approximately 18 hours after IONP injection, mice were euthanized using $\mathrm{CO}_{2}$ according to the IACUC approved protocol, followed by perfusion with $20 \mathrm{~mL}$ of PBS from the left ventricle. Fat, spleen, kidney, liver, and tumors growing on the peritoneal wall and in the peritoneal cavity were harvested in pre-weighed conical tubes. Each tissue was weighed and subjected to acid digestion using 3-10 mL of trace metal grade acid mixture $\left(9: 1 \mathrm{HNO}_{3}: \mathrm{HCl}\right.$; Thermo Fisher Scientific) at $90^{\circ} \mathrm{C}$ for 1 hour. A $0.5 \mathrm{~mL}$ aliquot of $30 \%$ trace metal $\mathrm{H}_{2} \mathrm{O}_{2}$ (GFC Chemicals, Powell, OH, USA) 
was then added, and the samples were diluted to $15-50 \mathrm{~mL}$ total volume with double-distilled water. Dartmouth Trace Element Analysis Core Facility measured each digest tissue's iron content using inductively coupled plasma mass spectrometry (ICP-MS).

In addition to quantitative iron analysis of tissues, qualitative histology was performed on representative tumor sections. Approximately 18 hours after IONP injection, mice were euthanized using $\mathrm{CO}_{2}$, and tumor cells on the peritoneal wall and cavity were harvested and embedded in paraffin. Paraffin sections $(5 \mu \mathrm{m})$ on microscope slides were heated in an oven set at $79^{\circ} \mathrm{C}$ for 30 minutes and deparafinized. Samples were stained for iron using a Gomori Prussian blue iron stain kit (Newcomer Supply, Middleton, WI, USA) following the manufacturer's protocol, and were then mounted with Permount ${ }^{\mathrm{TM}}$ (Thermo Fisher Scientific). Samples were immersed in hematoxylin (Thermo Fisher Scientific) for 5 minutes, washed with water, immersed in eosin (Fisher, Pittsburgh, PA) for 2 minutes, and washed with water again. Slides were dehydrated with increasing concentrations of ethanol and then xylene, and were mounted with Permount. Tissue histology was performed with the help of Dartmouth Pathology Translational Research Services.

\section{Results}

\section{Fab design, production, and analysis}

The workflow for IONP functionalization and subsequent conjugation with the engineered antibodies is shown in Figure 1. The two antibody fragments, Farletuzufab (Ffab), targeting the FOLR $\alpha$ cancer antigen, and Botulifab (Bfab), targeting the negative control protein botulinum toxin, were constructed such that each retained an unpaired C-terminal

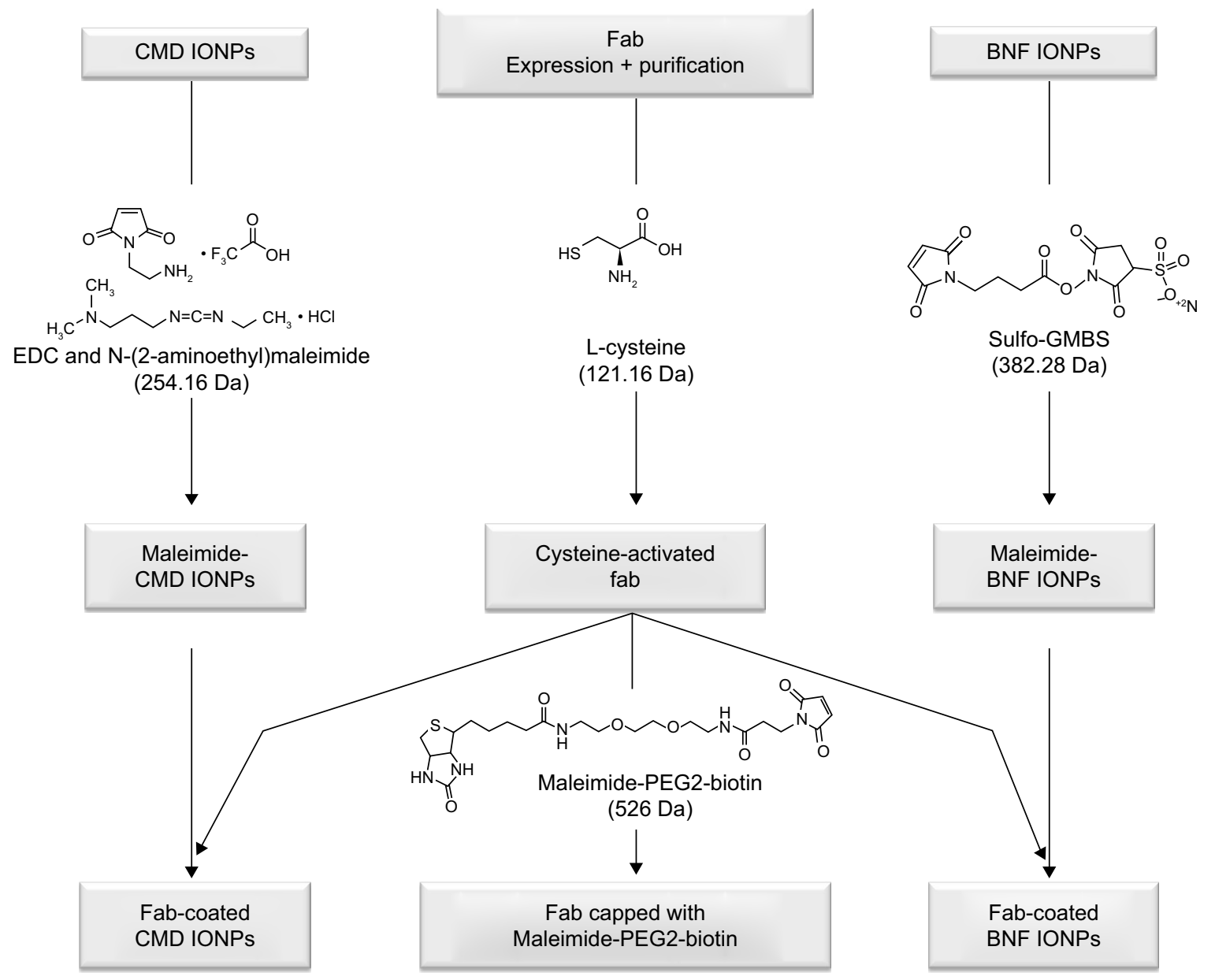

Figure I Schematic of workflow for fab and IONP functionalization.

Notes: Monomeric Ffab and Bfab are subjected to reduction/activation using $20 \mathrm{mM}$ cysteine followed by conjugation with maleimiede-PEG2-biotin or maleimide-activated IONPs. Two IONP types were examined in this study: Dartmouth CMD and commercial BNF, which were functionalized with maleimide groups using EDC and N-(2aminoethyl) maleimide, or sulfo-GMBS, respectively.

Abbreviations: IONP, iron oxide nanoparticle; fab, an engineered monoclonal antibody fragment; Ffab, Farletuzufab, engineered from monoclonal antibody Farletuzumab; Bfab, anti-botulinum toxin fab fragment; PEG2, polyethylene glycol 2; CMD, carboxymethyl-dextran; BNF, bionized nanoferrite; EDC, I-ethyl-3-(3-dimethylaminopropyl) carboiimide; sulfo-GMBS, $\mathrm{N}$-( $\gamma$-maleimobutyryloxy) sulfosuccinimide ester. 
cysteine on the heavy chain. This design ultimately enabled site-specific conjugation to either maleimide- $\mathrm{PEG}_{2}$-biotinor maleimide-functionalized IONPs. Ffab and Bfab were expressed in HEK 293 cells, and the desired fab monomers were isolated by Kappa select affinity purification followed by size exclusion chromatography (Figure S1A, B).

\section{Mass spectral analysis of Ffab and Bfab}

The identities of monomeric Ffab and Bfab were verified by liquid chromatography-mass spectrometry (LC-MS). The base peak of Ffab (expected [exp] =47,989 Da, observed [obs] $=48,109 \mathrm{Da}$ ) was $120 \mathrm{Da}$ heavier than expected, suggesting the presence of a cysteine adduct (Figure S1C). $\mathrm{Bfab}$, on the other hand, exhibited a +103 Da difference ( $\exp =48,528 \mathrm{Da}$, obs $=48,631 \mathrm{Da}$ ), the source of which was not immediately obvious (Figure S1D). Upon reductive activation with free cysteine, Ffab showed the expected peak at $47,989 \mathrm{Da}$ as well as peaks corresponding to free heavy chain (exp $=24,239 \mathrm{Da}$, obs $=24,239 \mathrm{Da})$ and free light chain $(\exp =23,750 \mathrm{Da}$, obs $=23,751 \mathrm{Da})$ (Figure S1E). Similar results were observed previously with a fab fragment of Trastuzumab, and it was determined then that the free heavy and light chains resulted from transient reduction of the intrachain disulfide bond, with no significant loss of binding activity or specificity. ${ }^{26}$ Cysteine-activated Bfab exhibited a -17 Da difference relative to the theoretical molecular mass (exp $=48,528 \mathrm{Da}$, obs $=48,511 \mathrm{Da})$. This difference is
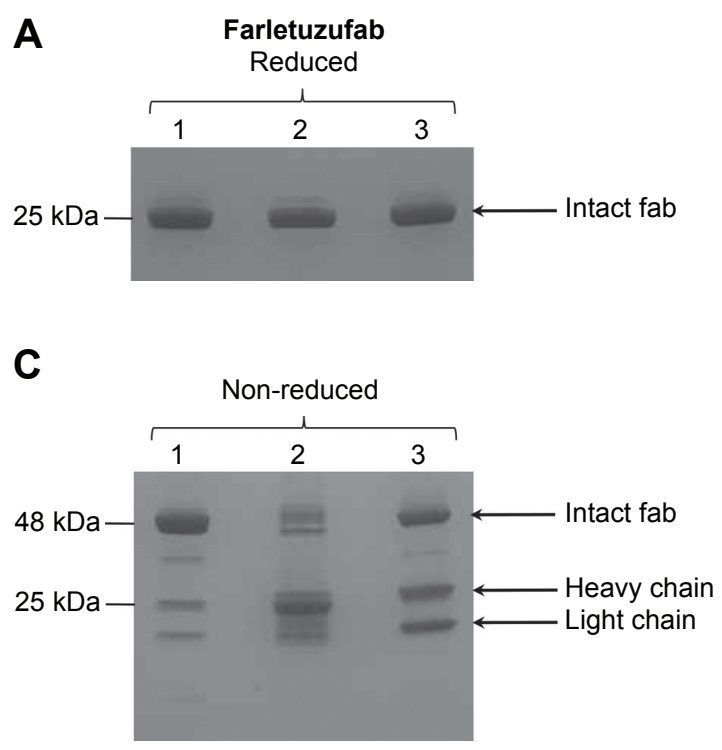

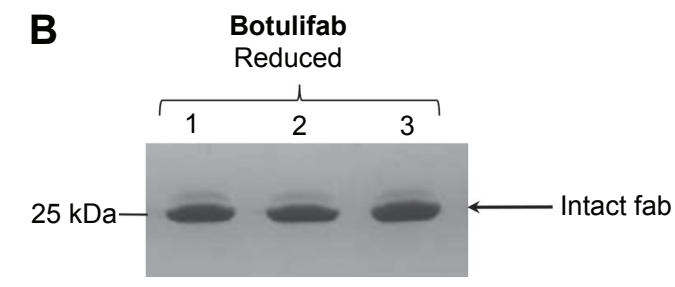

consistent with $\mathrm{N}$-terminal deamination of glutamine, ${ }^{28}$ and it explains the observed mass of the pre-activation material (ie, a de-aminated cysteine adduct yields +103 Da). Similar to cysteine-activated Ffab, the activated Bfab exhibited two lower molecular mass peaks that corresponded with free heavy and light chains (Figure S1F).

To assess the chemical reactivity of the engineered antibody fragments, conjugation reactions were performed with maleimide-PEG2-biotin. As expected, the reactions resulted in addition of one biotin moiety (+526 Da) to each of the intact fab antibodies (Figure S1G, H), and this observation demonstrated that the engineered fab was amenable to site-specific conjugation as designed. The free heavy chain and light chain of both activated antibody preparations also reacted as expected, with the addition of one biotin moiety to the light chain and two to the heavy chain. These results are also consistent with previous work on the Trastuzumab fab antibody fragment. ${ }^{26}$

Ffab and Bfab purities were evaluated by SDS-PAGE. Under reducing conditions (presence of $50 \mathrm{mM}$ dithiothreitol), the heavy and light chains of both Ffab and Bfab migrated as a single $25 \mathrm{kDa}$ band (Figure 2A, B; lane 1). In non-reducing conditions (no dithiothreitol), Ffab and Bfab migrated predominantly as the expected $48 \mathrm{kDa}$ band, although less intense low molecular weight bands corresponding to free heavy chain and light chain were also observed (Figure 2C, D; lane 1). Following reductive

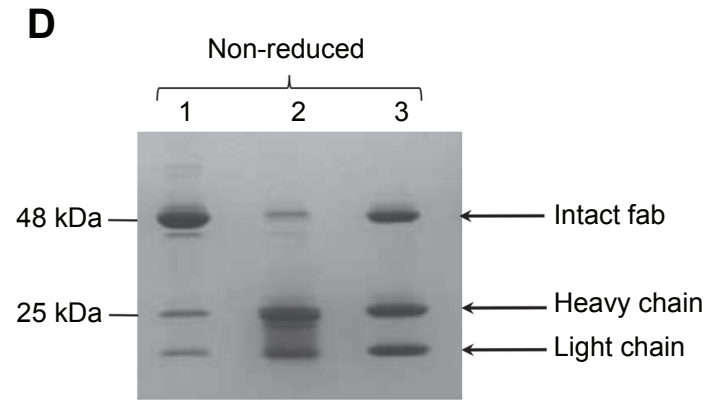

Figure 2 Characterization of Ffab and Bfab antibody fragments.

Notes: Reducing SDS-PAGE gels of (A) purified Ffab and (B) purified Bfab, stained with Coomassie brilliant blue. Non-reducing SDS-PAGE gel of (C) purified Ffab and (D) purified Bfab, stained with Coomassie brilliant blue. Lane I represents size exclusion-purified Ffab or Bfab, lane 2 is Ffab or Bfab after cysteine reductive activation, and lane 3 is Ffab or Bfab after maleimide-PEG2-biotin conjugation.

Abbreviations: SDS-PAGE, sodium dodecyl sulfate polyacrylamide gel electrophoresis; fab, an engineered monoclonal antibody fragment; Ffab, Farletuzufab, engineered from monoclonal antibody Farletuzumab; Bfab, Botulifab anti-botulinum toxin fab fragment; PEG2, polyethylene glycol 2. 
activation of both Ffab and Bfab, much of the intact fab band at $48 \mathrm{kDa}$ was shifted to the lower molecular weight bands for free heavy chain and light chain (Figure 2C, D; lane 2), which was consistent with the appearance of these species during LC-MS analysis. Upon conjugation to maleimide-PEG2-biotin, substantial portions of the intact fabs were reconstituted, although bands for free heavy and light chain remained evident (Figure 2C, D; lane 3). The LC-MS analysis had identified heavy and light chain species in which the cysteines involved in interchain disulfide bond formation had been capped with maleimide-PEG2biotin, and the corresponding free heavy and free light chain bands in the SDS-PAGE of biotinylated fabs corroborated that result (ie, maleimide-capped light and heavy chains are unable to reform intrachain disulfide bonds). We emphasize again that previous studies have shown that analogous, reduced yet intact fabs retain the binding activity and efficiency of their disulfide bonded counterparts. ${ }^{26,29}$

\section{Ffab binds specifically to FOLR $\alpha$}

Binding of Ffab and Bfab to recombinant FOLR $\alpha(\mathrm{rFOLR} \alpha)$ was initially analyzed by ELISA. The biotin-labeled Ffab fragment exhibited a half maximal effective concentration $\left(\mathrm{EC}_{50}\right)$ of $12 \mathrm{nM}$, whereas no binding was observed with the biotinylated Bfab control (Figure 3A). Additionally, more detailed $\mathrm{rFOLR} \alpha$ binding kinetics were analyzed by biolayer interferometry (Figure S2). Ffab-maleimide-PEG2-biotin was immobilized on streptavidin biosensor tips and assayed with $\mathrm{rFOLR} \alpha$ protein. The equilibrium dissociation constant for $\mathrm{Ffab}\left(\mathrm{K}_{\mathrm{D}}=14 \mathrm{nM}\right)$ was comparable to the $\mathrm{EC}_{50}$ obtained by ELISA (Figure 3A). Importantly, no detectable binding of
Bfab-maleiemide-PEG2-biotin was observed with $\mathrm{rFOLR} \alpha$ using the same streptavidin biosensors tips (data not shown). In aggregate, the quantitative binding studies with $\mathrm{rFOLR} \alpha$ demonstrated that the engineered $\mathrm{Ffab}$ retained good binding affinity to $\mathrm{rFOLR} \alpha$, to which the parental Farletuzumab $\operatorname{IgG}$ was found to have a $\mathrm{K}_{\mathrm{D}}=2 \mathrm{nM} \cdot{ }^{20}$

In addition to binding recombinant protein, it was critical that the engineered Ffab selectively bind the FOLR $\alpha$ surface protein in its native context. To assess cellular binding, the biotinylated Ffab and Bfab antibodies were assayed by flow cytometry using live human KB and SKBR3 cancer cells, where the former overexpress both FOLR $\alpha$ and human epidermal growth factor receptor 2 (HER2), and the latter overexpresses HER2 only. Ffab was found to exhibit high apparent binding affinity for $\mathrm{KB}$ cells $\left(\mathrm{EC}_{50}=13 \mathrm{nM}\right)$, whereas no substantial binding was observed on SKBR3 cells (Figure 3B). As expected, the negative control Bfab failed to bind either KB or SKBR3 tumor cells (Figure 3B). Thus, the Ffab antibody efficiently targeted cancer cells in a FOLR $\alpha$-dependent fashion.

\section{Analysis of Ffab-functionalized IONPs}

Having validated Ffab binding affinity and specificity for soluble and cell surface FOLR $\alpha$, the antibody fragment was conjugated to both CMD and BNF-maleimide-IONPs that differ in nanoscale structure and carbohydrate coating (Figure S3). These conjugations yielded Ffab-CMD and FfabBNF constructs that differed in size $(\sim 120 \mathrm{~nm}$ vs $\sim 190 \mathrm{~nm}$ hydrodynamic diameter, respectively) (Figure $\mathrm{S} 4$ ) and number of antibody-targeting moieties ( $\sim 50$ vs $\sim 500$ moieties, respectively; Table 1).
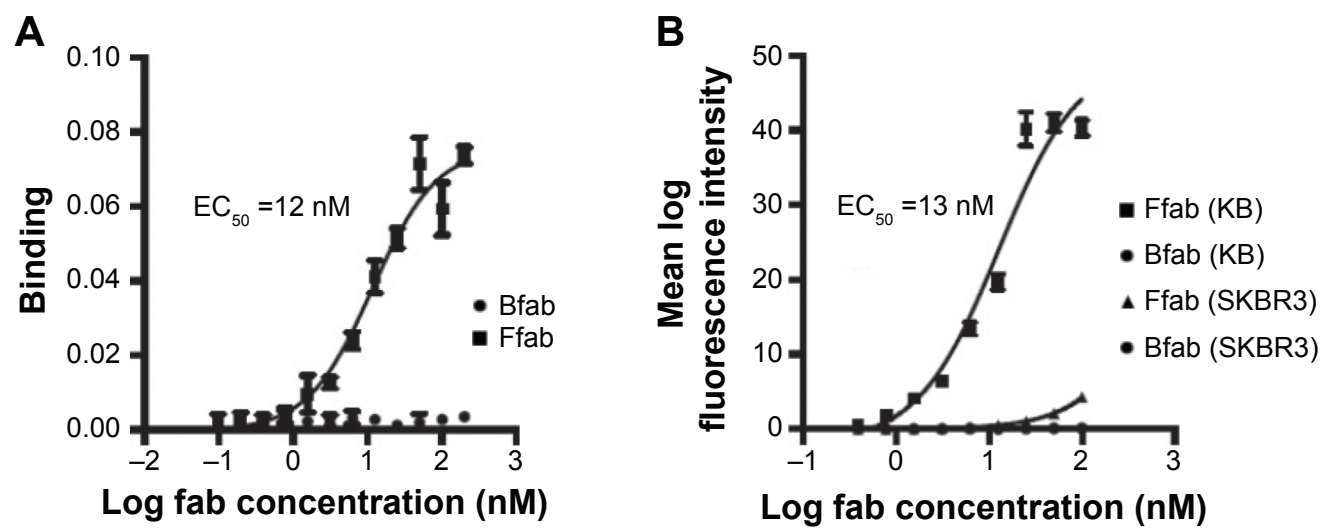

Figure 3 Binding analysis of Ffab and Bfab antibody fragments.

Notes: (A) Representative ELISA binding profile of Ffab (closed square) and Bfab (open circle) with rFOLR $\alpha$ protein. (B) Representative cell-based ELISA for Ffab (closed symbols) and Bfab (open symbols) binding to FOLR ${ }^{+} / \mathrm{HER} 2^{+} \mathrm{KB}$ cells (squares) or FOLR $/$ HER2 $2^{+}$SKBR3 cells (triangles).

Error bars represent standard deviation from technical triplicates.

Abbreviations: fab, an engineered monoclonal antibody fragment; Ffab, Farletuzufab, engineered from monoclonal antibody Farletuzumab; Bfab, Botulifab anti-botulinum toxin fab fragment; ELISA, enzyme-linked immunosorbent assay; FOLR, folate receptor; rFOLR $\alpha$, recombinant folate receptor alpha; HER2, human epidermal growth factor receptor 2. 
Table I Biophysical and biochemical characterization of IONP constructs

\begin{tabular}{lllll}
\hline Particle design & Hydrodynamic diameter $(\mathbf{n m})$ & PDI $^{\mathbf{a}}$ & Zeta potential $(\mathbf{m V})$ & Mean fab/lONPs \\
\hline CMD*-Mal & 115 & 0.146 & -0.320 & N/A \\
Bfab-CMD & 118 & 0.186 & -0.400 & 50 \\
Ffab-CMD & 120 & 0.230 & -0.141 & 50 \\
BNF*-Mal & 143 & 0.146 & -0.170 & $\mathrm{~N} / \mathrm{A}$ \\
Bfab-BNF & 190 & 0.400 & -0.500 & 500 \\
Ffab-BNF & 182 & 0.324 & -0.136 & 500 \\
\hline
\end{tabular}

Notes: aPolydispersity index; *CMD particles have a concentration of $0.5 \mathrm{mg}$ of iron/mg of particles, whereas BNF particles have $0.6 \mathrm{mg}$ of iron/mg of particles. Abbreviations: IONP, iron oxide nanoparticle; PDI, polydispersity index; N/A, not applicable; fab, an engineered monoclonal antibody fragment; Ffab, Farletuzufab, engineered from monoclonal antibody Farletuzumab; Bfab, Botulifab anti-botulinum toxin fab fragment; CMD, carboxymethyl-dextran; BNF, bionized nanoferrite; Mal, maleimide.

FOLR $\alpha$ binding of the Ffab-CMD and Ffab-BNF constructs, and their respective Bfab-IONP negative control counterparts was evaluated by ELISA (Figure 4A, B). Compared to the monomeric Ffab antibody, both Ffab-IONP constructs exhibited orders of magnitude higher apparent affinities $\left(\right.$ Ffab-BNF EC E0 $_{50}=0.013 \mathrm{nM}$; Ffab-CMD EC ${ }_{50}=0.16 \mathrm{nM}$ ), which reflects avidity effects derived from the nanoparticles' polyvalent nature. Conversely, no substantial binding was observed with the Bfab-BNF or Bfab-CMD negative control nanoparticles. These results demonstrated that $\mathrm{Ffab}$ binding of $\mathrm{rFOLR} \alpha$ was not compromised during IONP conjugation, and that the Bfab-IONP controls had no inherent affinity for the recombinant receptor. It is noteworthy that the larger Ffab-BNF particles exhibited 10-fold higher apparent affinity compared to the Ffab-CMD particles. This effect likely results from their 10 -fold difference in number
A
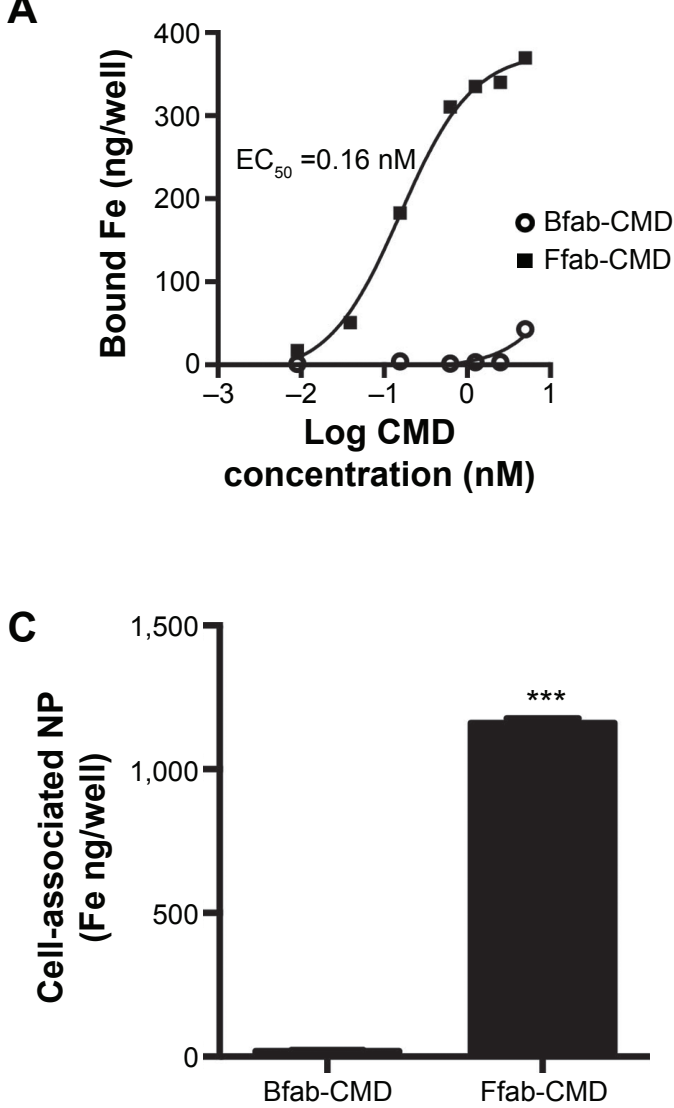

B
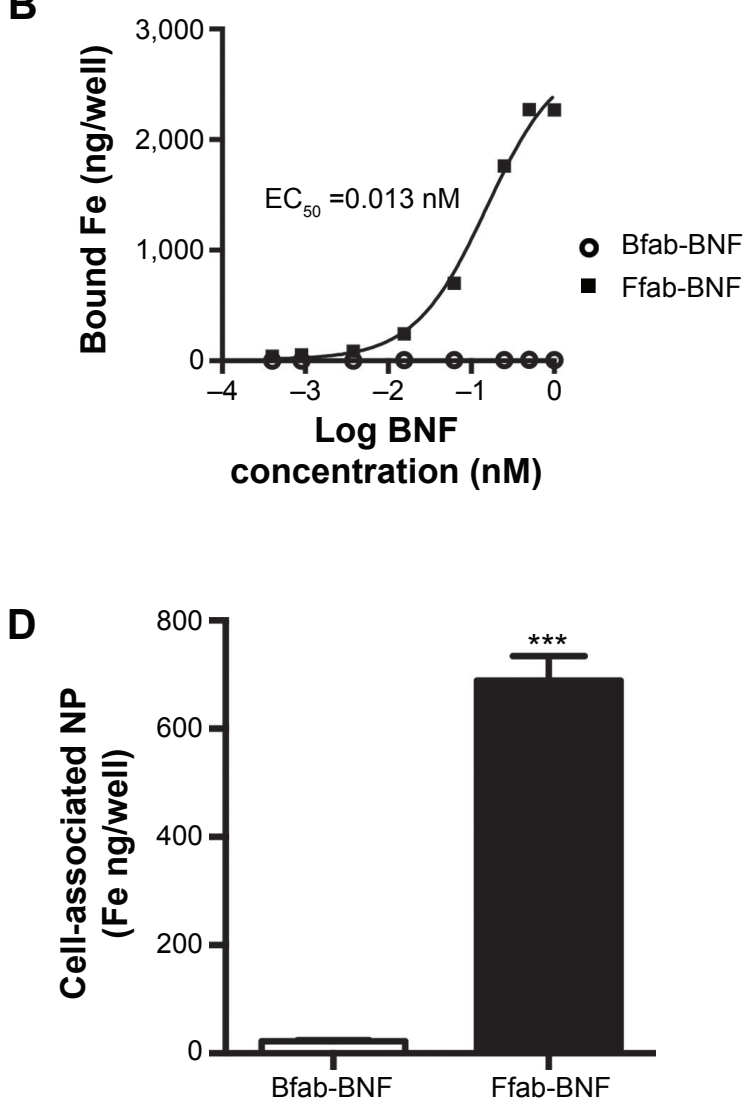

Figure 4 In vitro binding studies of Ffab-IONPs and Bfab-IONPs.

Notes: (A) Dose-response binding curves for Ffab-CMD (closed square) and Bfab-CMD (open circles) with rFOLR $\alpha$ protein. (B) Dose-response binding curves for Ffab$\mathrm{BNF}$ (closed square) and Bfab-BNF (open circles) with rFOLR $\alpha$ protein. (C) Binding of FOLR $\alpha^{+} \mathrm{KB}$ cancer cells by Ffab-CMD and Bfab-CMD dosed at $35 \mu \mathrm{g} / \mathrm{mL}(0.6 \mathrm{nM})$. (D) Binding of FOLR $\alpha^{+} \mathrm{KB}$ cancer cells by Ffab-BNF and Bfab-BNF dosed at $35 \mu \mathrm{g} / \mathrm{mL}(0.035 \mathrm{nM})$. Error bars represent standard deviation from technical triplicates. $* * * P<0.00 \mathrm{I}$, two-tailed unpaired $t$-test.

Abbreviations: fab, an engineered monoclonal antibody fragment; Ffab, Farletuzufab, engineered from monoclonal antibody Farletuzumab; Bfab, Botulifab anti-botulinum toxin fab fragment; IONP, iron oxide nanoparticle; CMD, carboxymethyl-dextran; rFOLR $\alpha$, recombinant folate receptor alpha; BNF, bionized nanoferrite, NP, nanoparticle. 
of conjugated antibodies (500 vs 50 antibodies for BNF and $\mathrm{CMD}$, respectively; Table 1). The higher maximum signal of the Ffab-BNF particles is likely due, in part, to their higher apparent affinity, and in part, due to the fact that BNF nanoparticles possess greater iron content than CMD nanoparticles $(0.6 \mathrm{mg}$ iron $/ \mathrm{mg}$ of particles vs $0.5 \mathrm{mg}$ iron $/ \mathrm{mg}$ of particles, respectively).

The targeting capacity of the nanoparticles was further assessed using FOLR $\alpha$-positive cancer cells. Both Ffab-CMD and Ffab-BNF efficiently bound adherent KB tumor cells during an 8-hour incubation in complete medium, whereas the Bfab-targeted IONPs showed little to no cellular association (Figure 4C, D). Importantly, the greater accumulation of Ffab-CMD $(0.6 \mathrm{nM})$ compared to Ffab-BNF $(0.035 \mathrm{nM})$ is due to the experimental design, where both particle types were incubated at a mass concentration of $35 \mu \mathrm{g} / \mathrm{mL}$, yielding a 17-fold greater molar concentration of CMD nanoparticles.

As part of the KB cellular binding studies, the subcellular localization of Ffab-CMD and Ffab-BNF conjugates was analyzed by transmission electron microscopy (TEM).
TEM micrographs showed that, following an 8-hour cellular incubation in vitro, Ffab-CMD and Ffab-BNF can be found accumulated on both the cell membrane as well as within intracellular vesicles (Figure 5A, B, D, E). Consistent with the negligible binding of Bfab-CMD and Bfab-BNF observed above (Figure 4C, D), no Bfab-targeted particles could be found in corresponding TEM images (Figure 5C, F). In aggregate, these results demonstrate specific and efficient targeting of FOLR $\alpha$-positive cancer cells by Ffab-CMD and Ffab-BNF, and additionally, they reveal that the Ffab-targeting moiety facilitates cellular internalization of both nanoparticle constructs.

\section{In vivo tumor targeting of IONPs}

Based on the in vitro performance of targeted Ffab-IONPs, we anticipated that these nanoparticle constructs might also exhibit enhanced tumor localization in vivo. To test this hypothesis, a single dose (750 $\mu \mathrm{g}$ of iron) of each IONP was administered intraperitoneally (IP) to NSG mice bearing peritoneal tumors derived from human KB cells. Approximately 18 hours post-injection, tumors, surrounding fat, and other
A

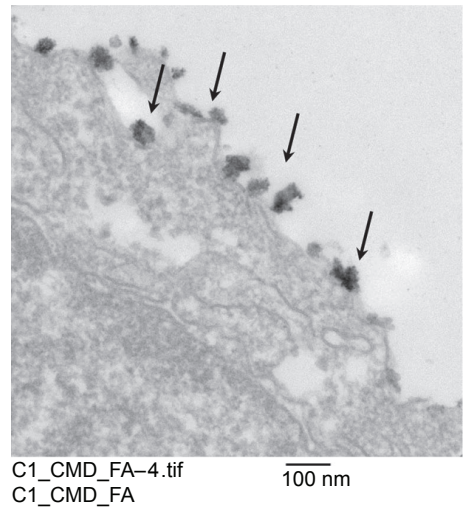

D

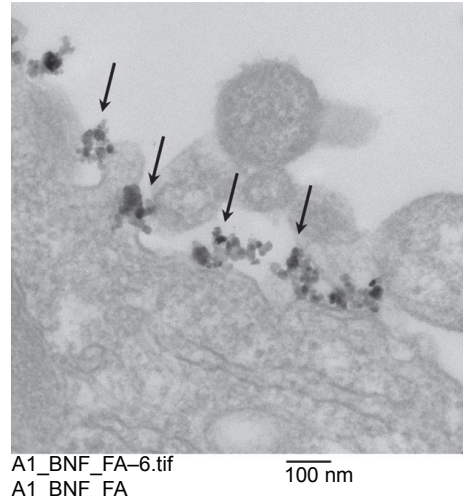

B

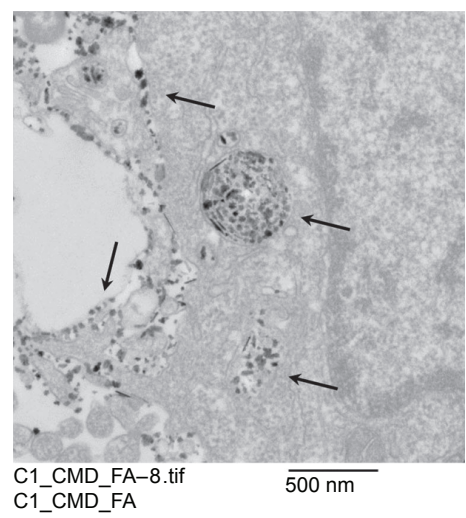

E

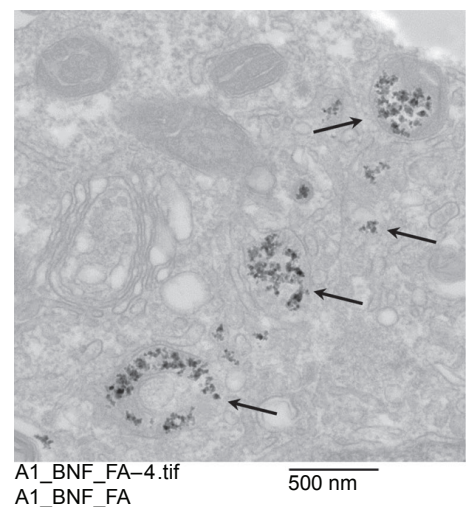

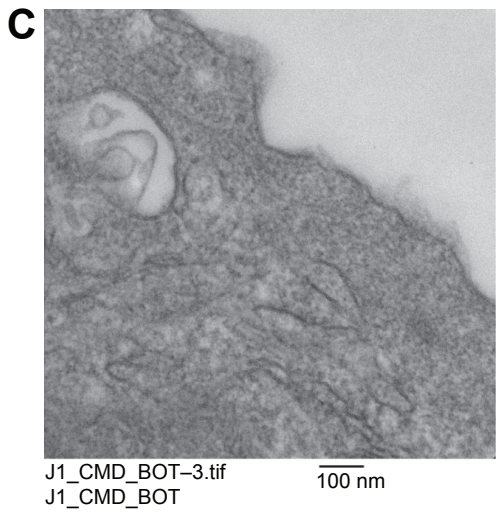

$\mathbf{F}$

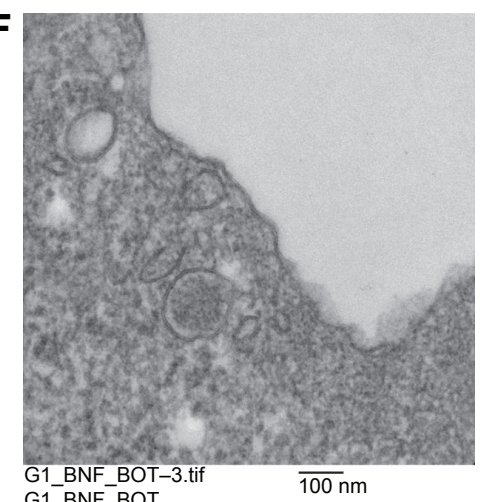

Figure 5 TEM imaging of Ffab-IONP and Bfab-IONP subcellular localization following in vitro binding to FOLR $\alpha^{+} \mathrm{KB}$ cancer cells.

Notes: Magnification of 25,000x showing the binding of (A) Ffab-CMD and (D) Ffab-BNF on the KB cell surface (arrows). Magnification of I0,000× showing (B) Ffab-CMD and (E) Ffab-BNF within intracellular vesicles (arrows). Magnification of 25,000x showing no evidence of nanoparticles on KB tumor cells treated with (C) Bfab-CMD or (F) Bfab-BNF. Scale bars are $100 \mathrm{~nm}$ (A, C, D and F) and $500 \mathrm{~nm}$ (B and E).

Abbreviations: TEM, transmission electron microscopy; fab, an engineered monoclonal antibody fragment; Ffab, Farletuzufab, engineered from monoclonal antibody Farletuzumab; Bfab, Botulifab anti-botulinum toxin fab fragment; IONP, iron oxide nanoparticle; CMD, carboxymethyl-dextran; rFOLR $\alpha$, recombinant folate receptor alpha; BNF, bionized nanoferrite; NP, nanoparticle. 
major organs in the abdominal cavity were harvested, and IONP content was quantified by ICP-MS (Figure 6 shows total tissue iron; Figure S5 shows tissue iron concentration). As seen in the in vitro cellular binding studies, the Bfab-CMD and Bfab-BNF negative controls failed to show significant iron accumulation within in vivo tumor masses (Figure 6A). In contrast, Ffab-BNF and Ffab-CMD yielded statistically significant increases in tumor-associated iron (Figure $6 \mathrm{~A}$ ), averaging $\sim 5 \%$ or $\sim 7 \%$, respectively, of the initially injected dose. Histology of tissue sections showed the $\mathrm{KB}$ tumors to be largely localized on the peritoneal wall (Figure 7A-C), and Prussian blue staining for iron was qualitatively consistent with the ICP-MS analysis, revealing significant iron accumulation on the surface of and within tumors from animals treated with Ffab-BNF (compare Figure $7 \mathrm{~F}$ to $\mathrm{D}$ and $\mathrm{E}$ ).

For both Ffab-IONPs and Bfab-IONPs, significant iron accumulation was also noted in the peritoneal fat tissue. There was a trend toward greater fat accumulation of Ffab-CMD vs the other constructs ( $\sim 3 \%$ of the injected dose vs $\sim 2 \%$ for other constructs), although the difference was only significant in comparison to Bfab-BNF (Figure 6B). For all four particle types, the majority of the recovered nanoparticles were found in the liver (Figure 6C). While the identity of the targeting antibody did not substantially influence liver accumulation, CMD-based nanoparticles showed a significantly higher liver concentration than did BNF nanoparticles $(\sim 40 \%$ injected dose vs $\sim 20 \%$, respectively). When compared to PBS-injected animals, animals treated with IONPs experienced no significant increase in splenic or kidney iron levels (Figure 6D and E, respectively).

\section{Discussion}

IONPs represent multi-functional nanomaterials with enormous value in the field of oncology. They have undergone extensive development as contrast agents for imaging, as cell capture reagents for diagnostics, as controlled release drug delivery platforms, and as energy converters for heat deposition in magnetic hyperthermia. ${ }^{7,9,10,30-54}$ In all of these applications, the ultimate utility of IONPs is critically dependent on differential association with malignant vs healthy cells; selective partitioning to the tumor environment and/or tumor cells is the key to enabling effective IONP diagnostics and therapeutics. In the current study, we have examined molecular targeting of IONPs to FOLR $\alpha$-positive cancers using an engineered antibody fragment.
A

Tumor
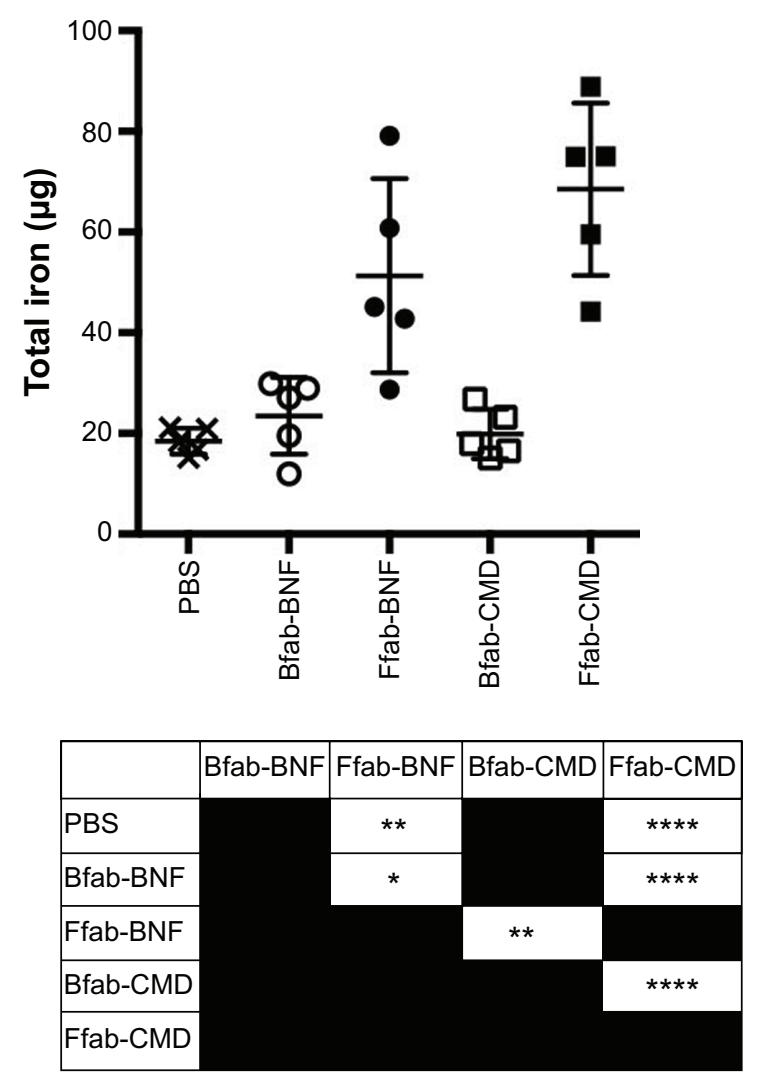

B
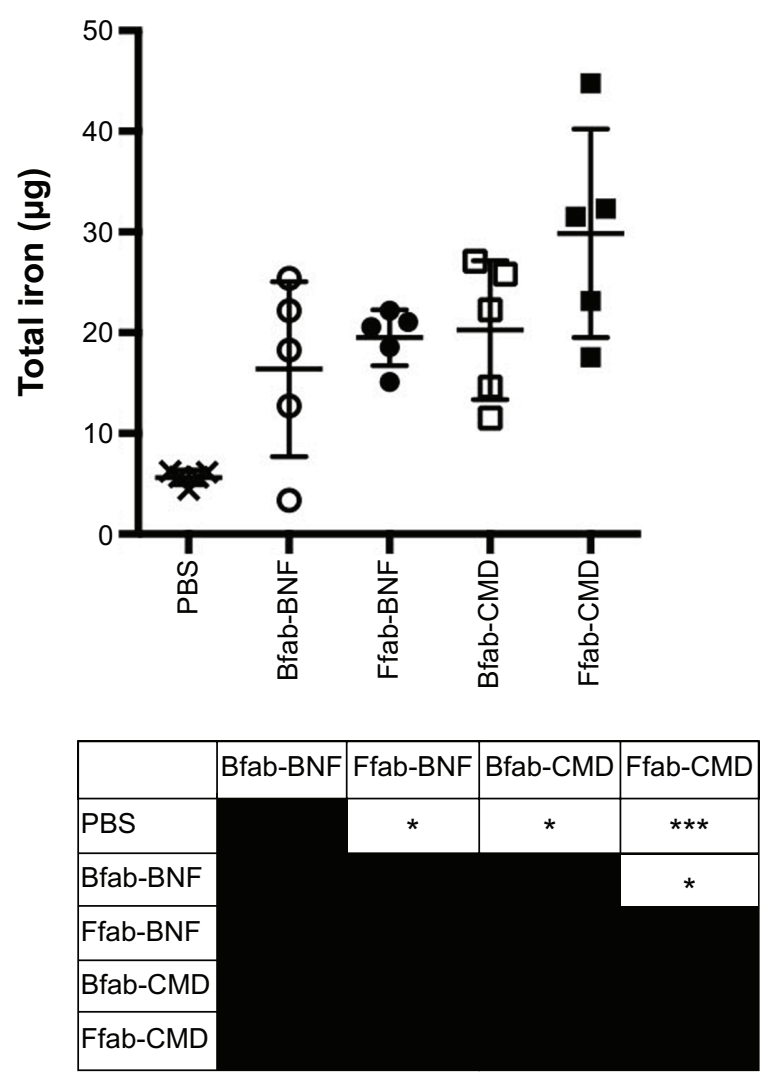

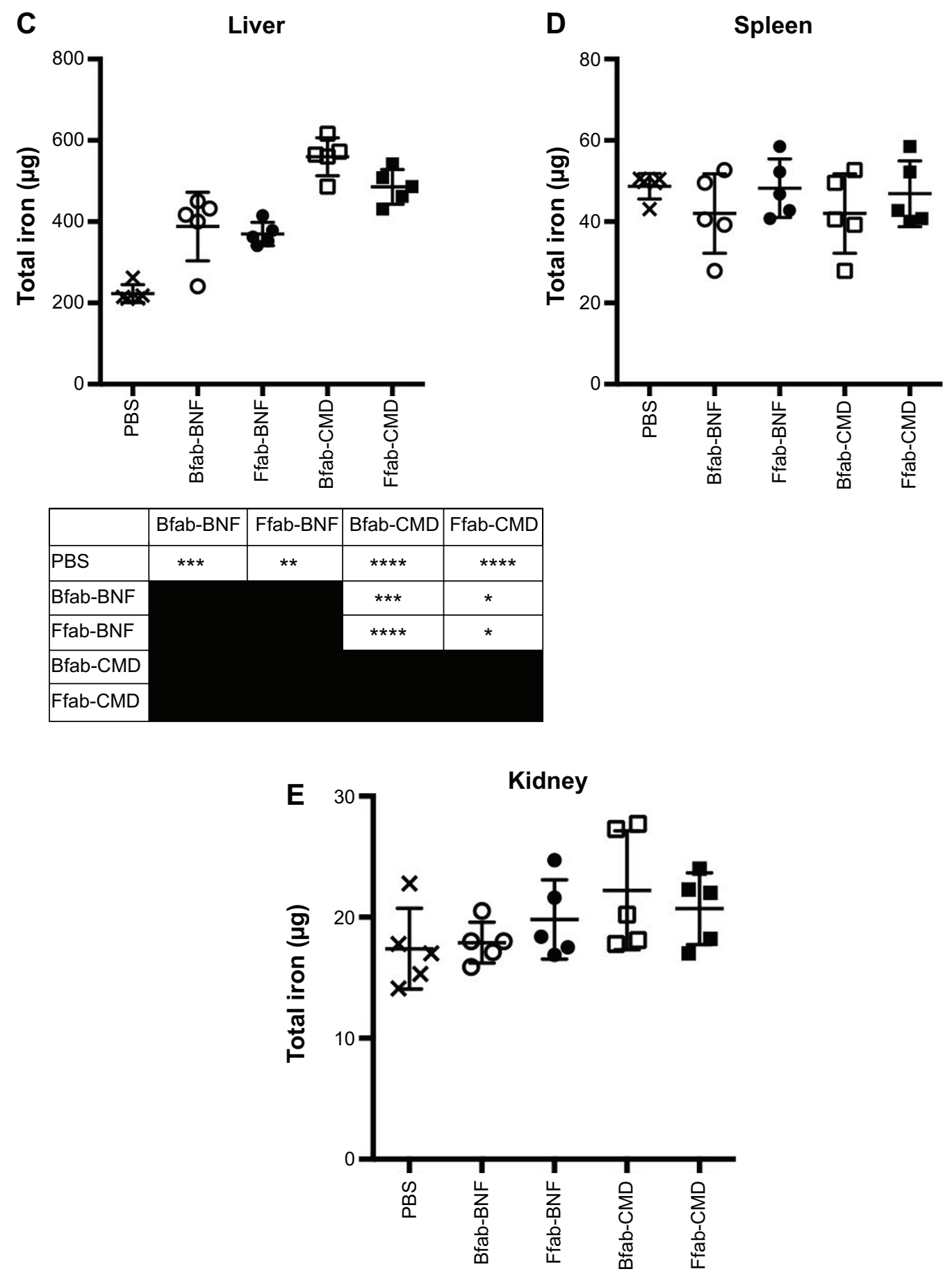

Figure 6 In vivo biodistribution of IONPs following IP administration.

Notes: Total iron content of various tissue compartments is shown: (A) tumor; (B) fat; (C) liver; (D) spleen; and (E) kidney. Data obtained by ICP-MS from five mice per group approximately 18 hours post-injection. Statistical significance was analyzed by one-way ANOVA with a Tukey multiple comparison post-test, and the results of individual comparisons are provided in the tables below each graph. $* * * * P<0.000 \mathrm{I} ; * * * P<0.00 \mathrm{I} ; * * \mathrm{P}<0.0 \mathrm{I} ; * \mathrm{P}<0.05$.

Abbreviations: PBS, phosphate-buffered saline; fab, an engineered monoclonal antibody fragment; Ffab, Farletuzufab, engineered from monoclonal antibody Farletuzumab; Bfab, Botulifab anti-botulinum toxin fab fragment; CMD, carboxymethyl-dextran; rFOLR $\alpha$, recombinant folate receptor alpha; BNF, bionized nanoferrite; IONPs, iron oxide nanoparticles; IP, intraperitoneal; ICP-MS, inductively coupled plasma mass spectrometry; ANOVA, analysis of variance.

FOLR $\alpha$ is a glycosylphosphatidylinositol-anchored glycoprotein whose expression is generally restricted in normal tissues, while often exhibiting high expression levels in cancers of epithelial origin. ${ }^{13,14}$ IONPs have been targeted to ovarian cancers via functionalization with folic acid, the natural ligand of FOLR $\alpha \cdot{ }^{15}$ Importantly, however, folate is an essential nutrient, and healthy cells acquire folate from their environment via numerous high-affinity membrane receptor proteins and transporters, including FOLR $\alpha$, FOLR beta, FOLR gamma ${ }^{19}$ reduced folate carrier (solute carrier 

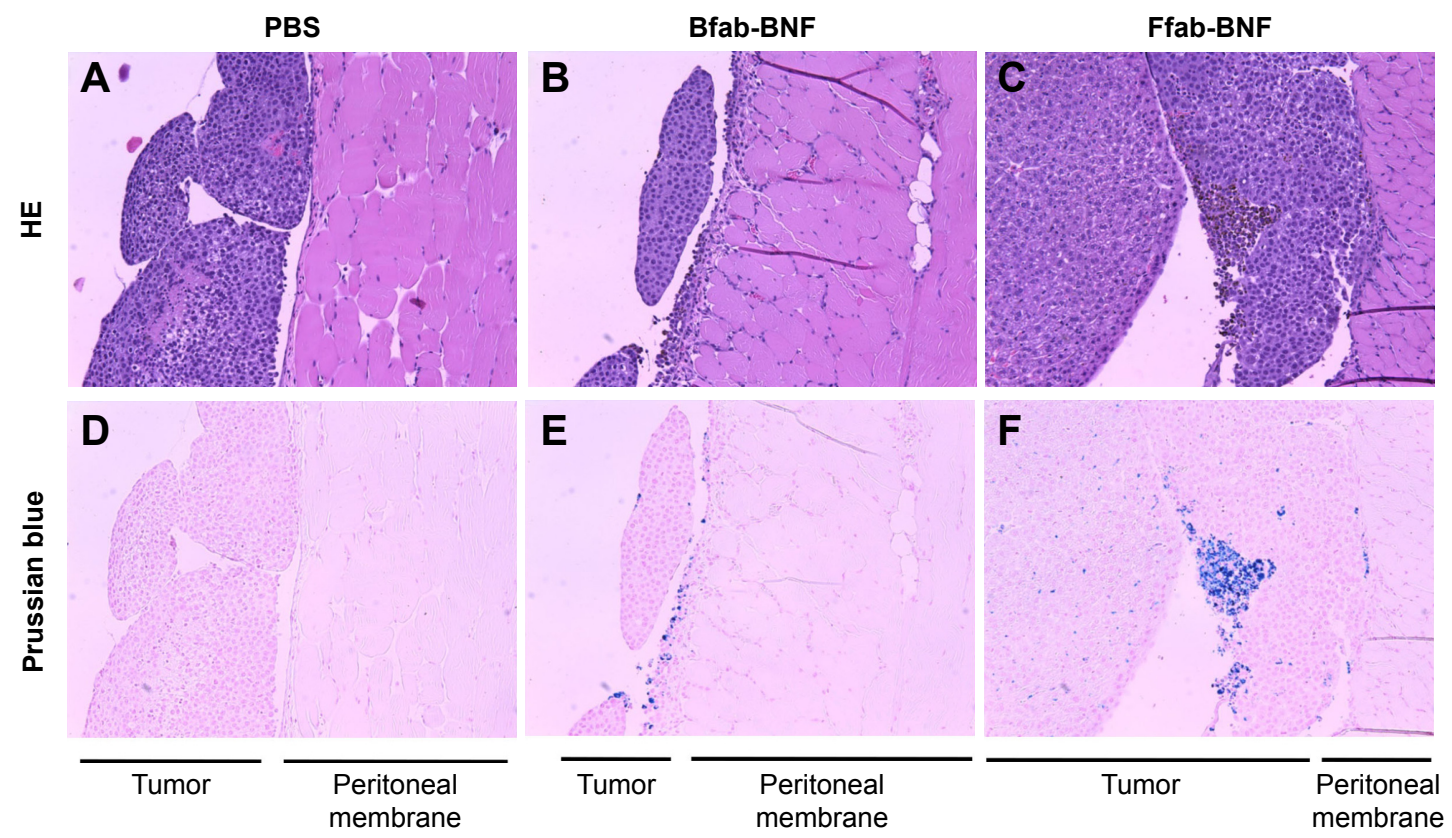

$\times 100$

Figure 7 Histology of excised tumors from ovarian cancer model.

Notes: Representative images where the tumor and peritoneal membrane are indicated. HE of tumor sections from animals treated with (A) PBS, (B) Bfab-BNF, or (C) Ffab-BNF. (D, E, F) Prussian blue staining of the same slides, respectively, to identify IONPs.

Abbreviations: HE, hematoxylin eosin; PBS, phosphate-buffered saline; fab, an engineered monoclonal antibody fragment; Ffab, Farletuzufab, engineered from monoclonal antibody Farletuzumab; Bfab, Botulifab anti-botulinum toxin fab fragment; IONPs, iron oxide nanoparticles; BNF, bionized nanoferrite.

19A1), ${ }^{55,56}$ and a proton-coupled folate transporter (solute carrier 46A1). ${ }^{57}$ Agents targeted by conjugation to folic acid can interfere with the uptake of naturally circulating folate, and they are known to bind both FOLR $\alpha$ and FOLR $\beta$, which reduce their selectivity for cancer cells. ${ }^{19}$ On the other hand, the monoclonal $\operatorname{IgG}$ antibody Farletuzumab is a highly specific binder of FOLR $\alpha$, and it has been shown to exhibit little to no cross-reactivity with healthy cells and tissues. ${ }^{20}$ Thus, Farletuzumab, or engineered fragments thereof, represent interesting candidates for tumor-specific targeting of IONPs.

In this study, we have demonstrated that 1) Ffab, a reformatted fragment of Farletuzumab, maintains the binding activity and specificity of the parental monoclonal antibody; and 2) functionalization of IONPs with Ffab substantially increases selective iron deposition in cultured cancer cells and in in vivo peritoneal tumors. Ffab-targeted IONPs were constructed from two different nanoparticle foundations: 1) commercially sourced BNF particles, and 2) custom synthesized CMD particles. The BNF particles had a larger hydrodynamic diameter $(\sim 190 \mathrm{~nm}$ vs $\sim 120 \mathrm{~nm})$, contained 1.2-fold more iron per particle, and bore a 10 -fold greater number of Ffab-targeting moieties on their surface.

The distinct characteristics of the two IONP platforms resulted in differential targeting efficiency in vitro, where the larger Ffab-BNF particles had a 10-fold higher affinity for rFOLR $\alpha$ and accumulated 10-fold more iron when used at an equivalent molar concentration. Conversely, when used at equal mass concentrations, Ffab-CMD particles accumulated approximately 2 -fold more iron during in vitro incubation with FOLR $\alpha$-positive cancer cells. Importantly, however, both Ffab-BNF and Ffab-CMD outperformed controls targeted to an irrelevant protein, and both Ffab-targeted IONP types resulted in substantial cellular internalization. Internalization of antibody-targeted IONPs has been reported previously. ${ }^{26,58,59}$ and the capacity to internalize IONPs via FOLR $\alpha$ could have important implications for hyperthermia or cytotoxic drug delivery to ovarian cancers. ${ }^{3,60}$

In contrast to their differential in vitro binding activities, in vivo administration of the two IONP platforms at a fixed iron dose showed no significant difference with respect to tumor homing in a murine model of ovarian cancer. This observation underscores the fact that, with respect to cancerspecific targeting, the results of in vitro experiments cannot be readily extrapolated to predict in vivo performance. In a previous murine model study of breast cancer, we showed that IONP size was the dominant determinant of tumor localization following intravenous administration of either targeted or non-targeted constructs. ${ }^{26}$ Interestingly, in that study, smaller $30 \mathrm{~nm}$ IONPs localized to the tumor 
compartment, whereas larger $100 \mathrm{~nm}$ IONPs did not. Conversely, larger $100 \mathrm{~nm}$ IONPs localized almost exclusively to the liver, whereas smaller $30 \mathrm{~nm}$ IONPs manifested significantly lower liver accumulation. In the current study, IONP size also correlated with liver sequestration, but in an inverted fashion: smaller $120 \mathrm{~nm}$ IONPs showed greater liver accumulation than larger $180 \mathrm{~nm}$ IONPs (40\% vs 20\% of the injected dose, respectively).

Unfortunately, numerous confounding factors make it difficult to draw meaningful conclusions from comparison of the current ovarian cancer model and the previous breast cancer model, ${ }^{26}$ (eg, different nanoparticle types, nanoparticle sizes, cancer cell lines, the nature of the models, the route of IONP administration, etc). Nonetheless, the results reported here provide important insights into the biodistribution of IONPs following IP administration. In particular, molecular targeting of FOLR $\alpha$ resulted in a significant tumor accumulation of IONPs. It bears noting that, in larger tumor cross-sections, IONPs exhibited highly concentrated accumulation in limited areas, but smaller, punctate accumulations were also seen distributed throughout the cross-sectional fields of view (Figure 7F). This non-uniform but tumor-wide deposition is more analogous to results seen with intravenous IONP administration than with direct tumor injection, where the former has recently been shown to achieve better efficacy in thermal ablation treatments. ${ }^{49}$ Specifically, in their work, Huang and Hainfeld note that uniform IONP distribution throughout the tumor is not required to achieve therapeutic heating, but rather tumor-wide deposition and encasement is adequate. ${ }^{49}$

The $5 \%-7 \%$ of initially injected IONP dose that was found in the tumor in the current study equaled that of a previous study that leveraged tumor-associated peritoneal phagocytes to localize non-targeted IONPs to ovarian tumor masses. ${ }^{6}$ It is unclear whether the NSG mouse model of the current study possesses similar tumor-associated peritoneal phagocytes, but the lack of any negative control Bfab-targeted IONPs in the tumor mass might suggest that phagocyte-mediated trafficking of IONPs to the tumor is compromised in the NSG model. If true, we speculate that the use of FOLR $\alpha$-targeted IONPs in a suitable immuno-competent model might result in additive tumor homing, and such a substantial IONP tumor accumulation would bode well for potential diagnostic and therapeutic applications.

In summary, in the present study, we have demonstrated molecular targeting of IONPs to the FOLR $\alpha$ cancer marker. Our targeting agent is based upon a clinically validated monoclonal antibody that has exquisite specificity for
FOLR $\alpha$ vs other folate receptors and transporters, and this selectivity may enable enhanced tumor accumulation relative to alternative FOLR $\alpha$ targeting strategies. These FOLR $\alpha$-specific iron oxide nanomaterials may ultimately prove useful in advancing diagnosis, imaging, and therapy of ovarian cancers.

\section{Acknowledgments}

The authors would like to thank Louisa Howard and Charles P Daghlian of the Dartmouth Electron Microscope Facility for their assistance with TEM data, and the Dartmouth Center for Cancer Nanotechnology Excellence Toxicology, Biodistribution and Pathology Core, and the Dartmouth Trace Element Core for ICP-MS data. We would also like to thank the Dartmouth Transgenic and Genetic Construct Shared Resources, and Jennifer Fields for maintaining the NSG mice. We also thank the Dartmouth CCOP Pathology Translational Research (especially Rebecca O’Meara and Eric York), for the assistance with tissue histology. Finally, we would like to thank Adimab LLC, in particular Yingda $\mathrm{Xu}$ and Felicia Reid, for their assistance with LC-MS data. This work was supported by NCI grant number 1 U54 CA151662-01.

\section{Disclosure}

The authors report no conflicts of interest in this work.

\section{References}

1. Howlader N, Noone AM, Krapcho M, et al. SEER Cancer Statistics Review, 1975-2011, National Cancer Institute. Bethesda, MD: National Cancer Institute; 2014. Available from: http://seer.cancer.gov/ csr/1975_2011/. Accessed November 27, 2014.

2. Zee Vd. Heating the patient: a promising approach. Annals of Oncology. 2002;13:1173-1184.

3. Moroz P, Jones SK, Gray BN. Magnetically mediated hyperthermia: current status and future directions. Int J Hyperthermia. 2002;18: 267-284.

4. Ito A, Shinkai M, Honda H, Wakabayashi T, Yoshida J, Kobayashi T. Augmentation of MHC class I antigen presentation via heat shock protein expression by hyperthermia. Cancer Immunol Immunother. 2001; 50:515-522.

5. Ito A, Shinkai M, Honda $\mathrm{H}$, et al. Heat shock protein 70 expression induces an antitumor immunity during intracellular hyperthermia using magnetite nanoparticles. Cancer Immunol Immunother. 2003;52: 80-88.

6. Toraya-Brown S, Sheen MR, Baird JR, et al. Phagocytes mediate targeting of iron oxide nanoparticles to tumors for cancer therapy. Integr Biol (Camb). 2013;5(1):159-171.

7. Gilchrist RK, Medal R, Shorey WD, Hanselman RC, Parrott JC, Taylor CB. Selective inductive heating of lymph nodes. Ann Surg. 1957;146: 596-606.

8. Ivkov R, DeNardo SJ, Daum W, et al. Application of high amplitude alternating magnetic fields for heat induction of nanoparticles localized in cancer. Clin Cancer Res. 2005;11:7093S-7103S.

9. Ito A, Shinkai M, Honda H, Kobayashi T. Medical application of functionalized magnetic nanoparticles. J Biosci Bioeng. 2005;100:1-11. 
10. Dennis CL, Jackson AJ, Borchers JA, et al. Nearly complete regression of tumors via collective behavior of magnetic nanoparticles in hyperthermia. Nanotechnology. 2009;20(39):395103.

11. Fortin JP, Wilhem C, Servais J, Ménager C, Bacri JC, Gazeau F. Size-sorted anionic iron oxide nanomagnets as colloidal mediators for magnetic hyperthermia. J Am Chem Soc. 2007;129(9):2628-2635.

12. Taratula O, Dani RK, Schumann C, et al. Multifunctional nanomedicine platform for concurrent delivery of chemotherapeutic drugs and mild hyperthermia to ovarian cancer cells. Int J Pharm. 2013;458:169-180.

13. Weitman SD, Lark RH, Coney LR, et al. Distribution of the folate receptor GP38 in normal and malignant cells lines and tissues. Cancer Res. 1992;52:3396-3401.

14. Garin-Chesa P, Campbell I, Saigo PE, Lewis JL Jr, Old LJ, Rettig WJ. Trophoblast and ovarian cancer antigen LK26. Sensitivity and specificity in immunopathology and molecular identification as a folate-binding protein. Am J Pathol. 1993;142:557-567.

15. Krais A, Wortmann L, Hermanns L, et al. Targeted uptake of folic acidfunctionalized iron oxide nanoparticles by ovarian cancer cells in the presence but not in the absence of serum. Nanomedicine. 2014;10(7): 1421-1431.

16. Quan G, Du X, Huo T, et al. Targeted molecular imaging of antigen OC183B2 in ovarian cancers using MR molecular probes. Acad Radiol. 2010;17:1468-1476.

17. Prantner AM, Nguyen CV, Scholler N. Facile immunotargeting of nanoparticles against tumor antigens using site-specific biotinylated antibody fragments. J Biomed Nanotechnol. 2013;9(10):1686-1697.

18. Kamen BA, Smith AK. Farletuzumab, an anti-folate receptor alpha antibody, does not block binding of folate or anti-folates to receptor nor does it alter the potency of anti-folates in vitro. Cancer Chemother Pharmacol. 2012;70(1):113-120.

19. Marchetti C, Palaia I, Giorgini M, et al. Targeted drug delivery via folate receptors in recurrent ovarian cancer: a review. Onco Targets Ther. 2014;7:1223-1236.

20. Ebel W, Routhier EL, Foley B, et al. Preclinical evaluation of MORAb003, a humanized monoclonal antibody antagonizing folate receptoralpha. Cancer Immun. 2007;7:6

21. Spannuth WA, Lyn YG, Merritt WM, et al. Therapeutic efficacy of folate receptor alpha blockade with MORAb-003 in ovarian cancer. Gynecol Oncol. 2008;108:S135.

22. Spannuth WA, Sood AK, Coleman RL. Farletuzumab in epithelial ovarian carcinoma. Expert Opin Biol Ther. 2010;10(3):431-437.

23. Armstrong DK, White AJ, Weil SC, Phillips M, Coleman RL. Farletuzumab (a monoclonal antibody against folate receptor alpha) in relapsed platinum-sensitive ovarian cancer. Gynecol Oncol. 2013;129:452-458.

24. Wallace TP, Harris WJ, Carr FJ, Rettig WJ, Garin-Chesa P, Lloyd J, inventors. Sloan-Kettering Cancer Center, assignee. Recombinant human anti-LK26 antibodies. United States patent US 5952484. 1999 Sept 14.

25. Marks JD, Amersdorfer P, Geren I, Lou J, Razai A, Consuelos MG, inventors. The Regents Of The University Of California, assignee. Therapeutic monoclonal antibodies that neutralize botulinum neurotoxins. United States patent US 20080124328A1. 2008 May 29.

26. Ndong C, Tate JA, Kett WC, et al. Tumor cell targeting by iron oxide nanoparticles is dominated by different factors in vitro versus in vivo. PLoS One. 2015;10(2):e0115636.

27. Kekalo KA, Baker I, inventors; Engineering DCTSo, assignee. Magnetic nanoparticles, composites, suspensions and colloids with high specific absorption rate (SAR). United States patent WO 2014089464 A1. 2014 Jun 12.

28. Liu H, Gaza-Bulseco G, Faldu D, Chumsae C, Sun J. Heterogeneity of monoclonal antibodies. J Pharm Sci. 2008;97(7):2426-2447.

29. Humphreys D, Heywood SP, Henry A, et al. Alternative antibody Fab' fragment PEGylation strategies: combination of strong reducing agents, disruption of the interchain disulphide bond and disulphide engineering. Protein Eng Des Sel. 2007;20(5):227-234.

30. Gupta AK, Gupta M. Synthesis and surface engineering of iron oxide nanoparticles for biomedical applications. Biomaterials. 2005;26: 3995-4021.
31. Kohler N, Sun C, Wang J, Zhang M. Methotrexate-modified superparamagnetic nanoparticles and their intracellular uptake into human cancer cells. Langmuir. 2005;21:8858-8864.

32. Lee H, Lee E, Kim DK, Jang NK, Jeong YY, Jon S. Antibiofouling polymer-coated superparamagnetic iron oxide nanoparticles as potential magnetic resonance contrast agents for in vivo cancer imaging. $\mathrm{J} \mathrm{Am}$ Chem Soc. 2006;128:7383-7389.

33. van Vlerken LE, Amiji MM. Multi-functionnal polymeric nanoparticles for tumor-targeted drug delivery. Expert Opin Drug Deliv. 2006; $3(2): 205-216$.

34. Lee JH, Huh YM, Jun YW, et al. Artificially engineered magnetic nanoparticles for ultra-sensitive molecular imaging. Nat Med. 2007; 13(1):95-99.

35. Peng X-H, Qian X, Mao H, et al. Targeted magnetic iron oxide nanoparticles for tumor imaging and therapy. Int J Nanomedicine. 2008;3(3): 311-321.

36. Cole AJ, Yang VC, David AE. Cancer theranostics: the rise of targted magnetic nanoparticles. Trends Biotechnol. 2011;29(7):323-332.

37. Laurent S, Mahmoudi M. Superparamagnetic iron oxide nanoparticles: promises for diagnosis and treatment of cancer. Int J Mol Epidemiol Genet. 2011;2(4):367-390.

38. Tassa C, Shaw SY, Weissleder R. Dextran-coated iron oxide nanoparticles: a versatile platform for targeted molecular imaging, molecular diagnostics, and therapy. Acc Chem Res. 2011;44(10):842-852.

39. Kaaki K, Hervé-Aubert K, Chiper M, et al. Magnetic nanocarriers of doxorubicin coated with poly(ethylene glycol) and folic acid: Relation between coating strucutre, surface properties, colloidal stability and cancer cell targeting. Langmuir. 2012;28:1496-1505.

40. Rosen JE, Chan L, Shieh DB, Gu FX. Iron oxide nanoparticles for targeted cancer imaging and diagnostics. Nanomedicine. 2012;8: 275-290.

41. Ittrich H, Peldschus K, Raabe N, Kaul M, Adam G. Superparmagnetic iron oxide nanoparticles in biomedicine: applications and developments in diagnostics and therapy. Rofo. 2013;185:1149-1166.

42. Kirui DK, Khalidov I, Wang Y, Batt CA. Targeted near-IR hybrid magnetic nanoparticles for in vivo cancer therpay and imaging. Nanomedicine. 2013;9:702-711.

43. Thomas R, Park I-K, Jeong YY. Magnetic iron oxide nanoparticles for multimodal imaging and therapy of cancer. Int J Mol Sci. 2013;14: 15910-15930.

44. Yen SK, Padmanabhan P, Selvan ST. Multifunctional iron oxide nanoparticles for diagnostics, therapy and macromolecule delivery. Theranostics. 2013;3(12):986-1003.

45. Cortajarena AL, Ortega D, Ocampo SM, et al. Engineering Iron oxide nanoparticles for clinical settings. Nanobiomed. 2014;1(2):58841.

46. Wabler M, Zhu W, Hedayati M, et al. Magnetic resonance imaging contrast of iron oxide nanoparticles developed for hyperthermia is dominated by iron content. Int J Hyperthermia. 2014;30(3):192-200.

47. Silva AC, Oliveira TR, Mamami JB, et al. Application of hyperthermia induced by superparamagnetic iron oxide nanoparticles in glioma treatment. Int J Nanomedicine. 2011;6:591-603.

48. Hilger I. In vivo applications of magnetic nanoparticles hyperthermia. Int J Hyperthermia. 2013;29(8):828-834.

49. Huang HS, Hainfeld JF. Intravenous magnetic nanoparticles cancer hyperthermia. Int J Nanomedicine. 2013;8:2521-2532.

50. Ivkov R. Magnetic nanoparticles hyperthermia: a new frontier in biology and medicine. Int J Hyperthermia. 2013;29(8):703-705.

51. Petryk AA, Giustini AJ, Gottesman RE, Kaufman PA, Hoopes PJ. Magnetic nanoparticle hyperthermia enhancement of cisplatin chemotherapy cancer treatment. Int J Hyperthermia. 2013;29:845-851.

52. Petryk AA, Giustini AJ, Gottesman RE, Trembly BS, Hoopes PJ. Comparison of magnetic nanoparticles and microwave hyperthermia cancer treatment methology and treatment effect in a rodent breast cancer model. Int J Hyperthermia. 2013;29:819-827.

53. Toraya-Brown S, Sheen MR, Zhang P, et al. Local hyperthermia of tumors induces $\mathrm{CD} 8(+) \mathrm{T}$ cell-mediated resistance against distal and secondary tumors. Nanomedicine. 2014;10(6):1273-1285. 
54. Kossatz S, Ludwig R, Dähring H, et al. High therapeutic efficiency of magnetic hyperthermia in xenograft models achieved with moderate temperature dosages in tumor area. Pharm Res. 2014;31:3274-3288.

55. Marthely LH, Hou Z, Deng Y. Human reduced folate carrier: translation of basic biology to cancer etiology and therapy. Cancer Metastasis Rev. 2007;26(1):111-128.

56. Marthely LH, Hou Z. Structure and function of the reduced folate carrier: a paradigm of a major facilitator superfamily mammalian nutrient transporter. Vitam Horm. 2008;79:145-184.

57. Qiu A, Jansen M, Sakaris A, et al. Identification of an intestinal folate transporter and the molecular basis for hereditary folate malabsorption. Cell. 2006;127(5):917-928.
58. Hilger I, Dietmar E, Linß W, Strec S, Kaiser WA. Developments for the minimally invasive treatment of tumours by targted magnetic heating. J Phys: Condens Mat. 2006;18:S2951-S2958.

59. Zhang JP, Dewilde AH, Chinn P, et al. Herceptin-directed nanoparticles activated by an alternating magnetic field slectively kill HER-2 positive human breast cells in vitro via hyperthermia. Int J Hyperthermia. 2011;27:682-697.

60. Wahajuddin, Arora S. Superparamagnetic iron oxide nanoparticles: magnetic nanoplatforms as drug carriers. Int J Nanomedicine. 2012;7 3445-3471. 


\section{Supplementary materials}
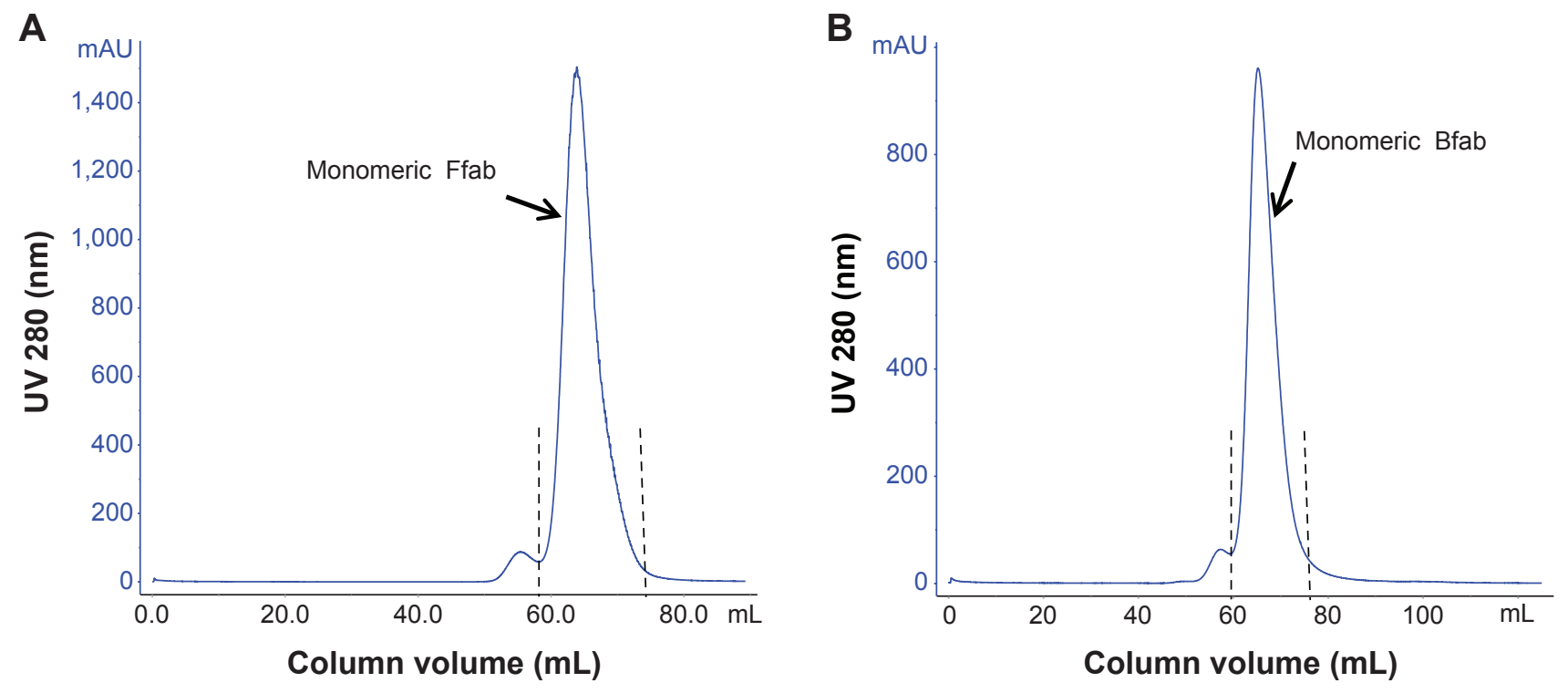

C

Ffab (SEC)

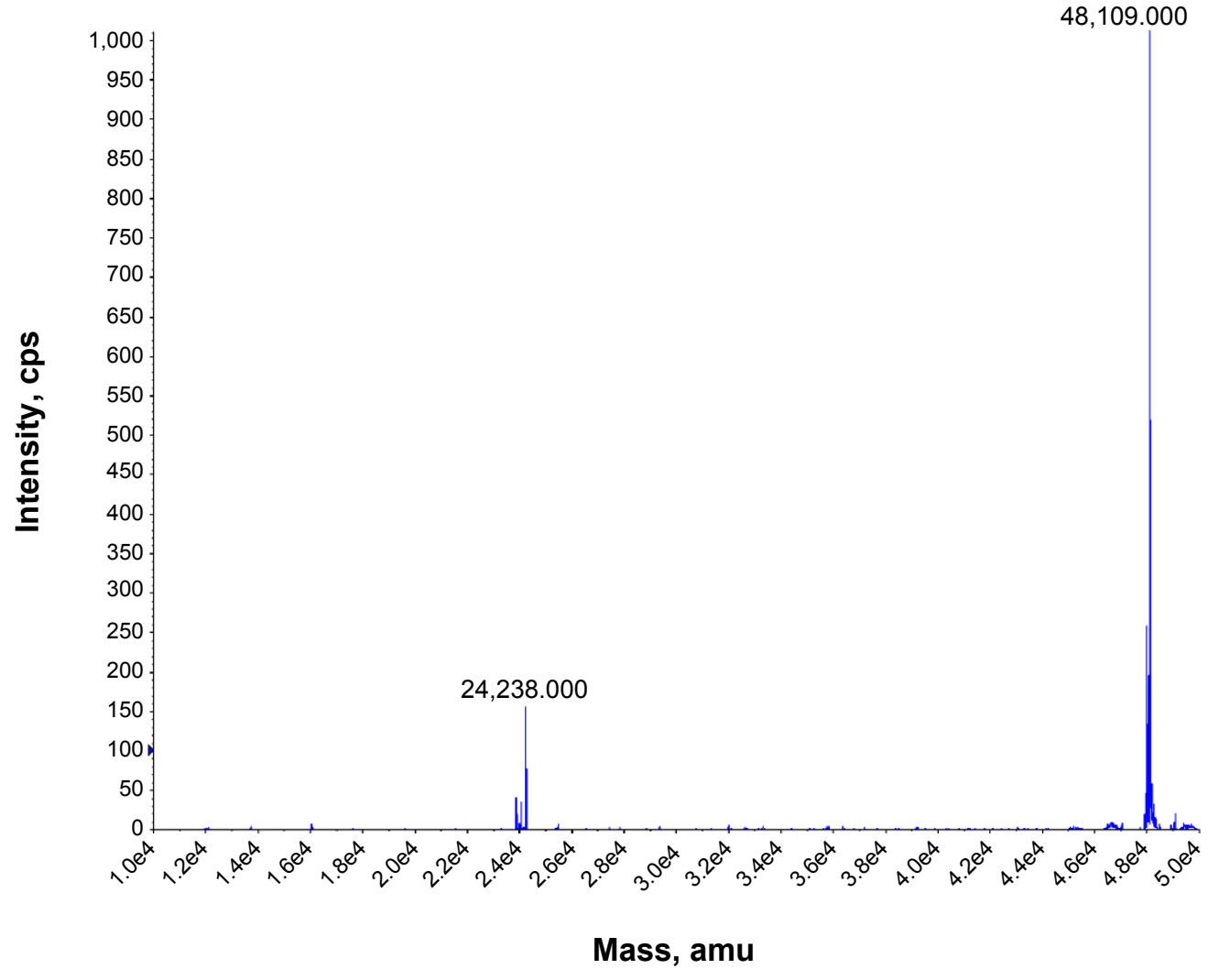

Figure SI (Continued) 
D

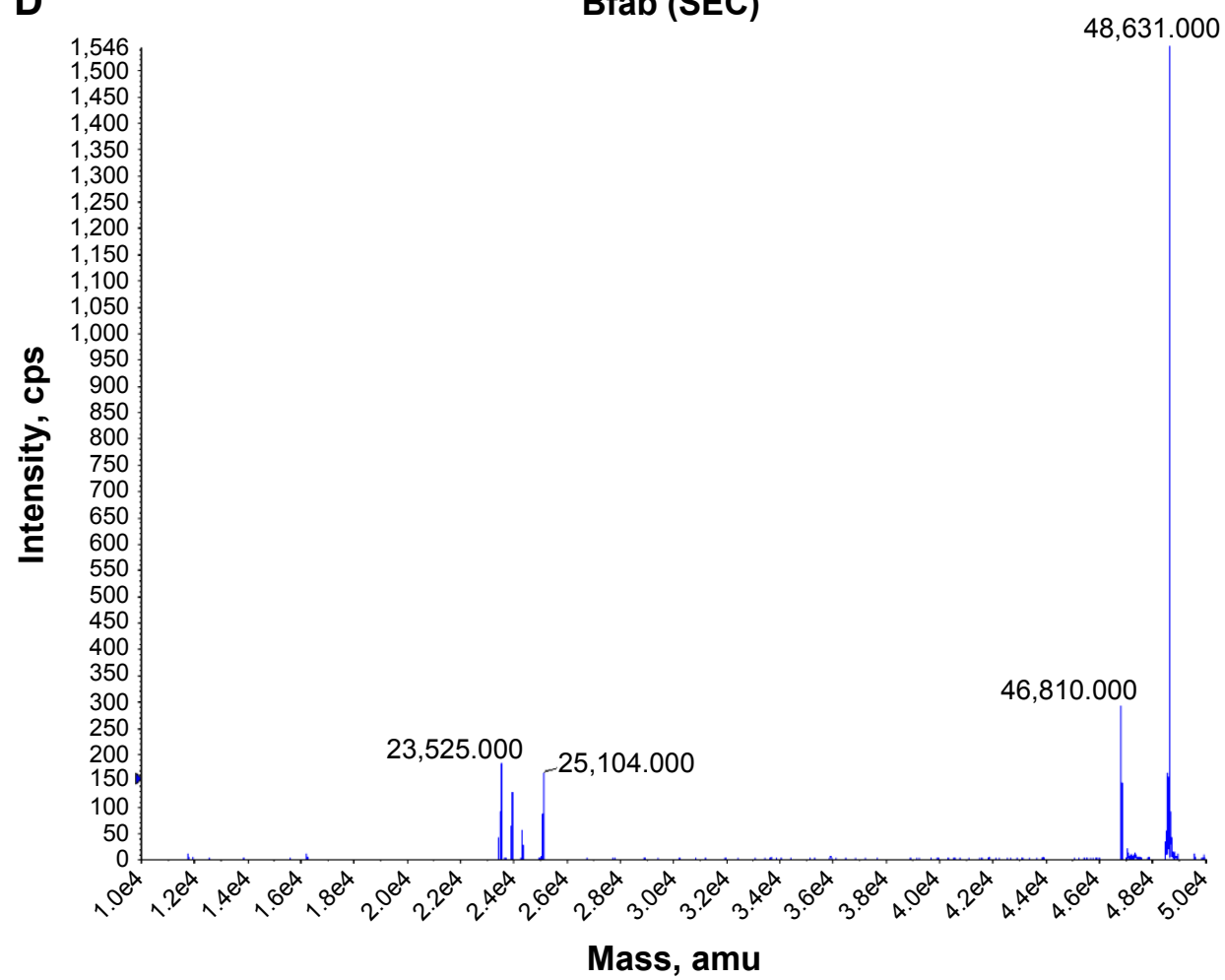

$\mathbf{E}$

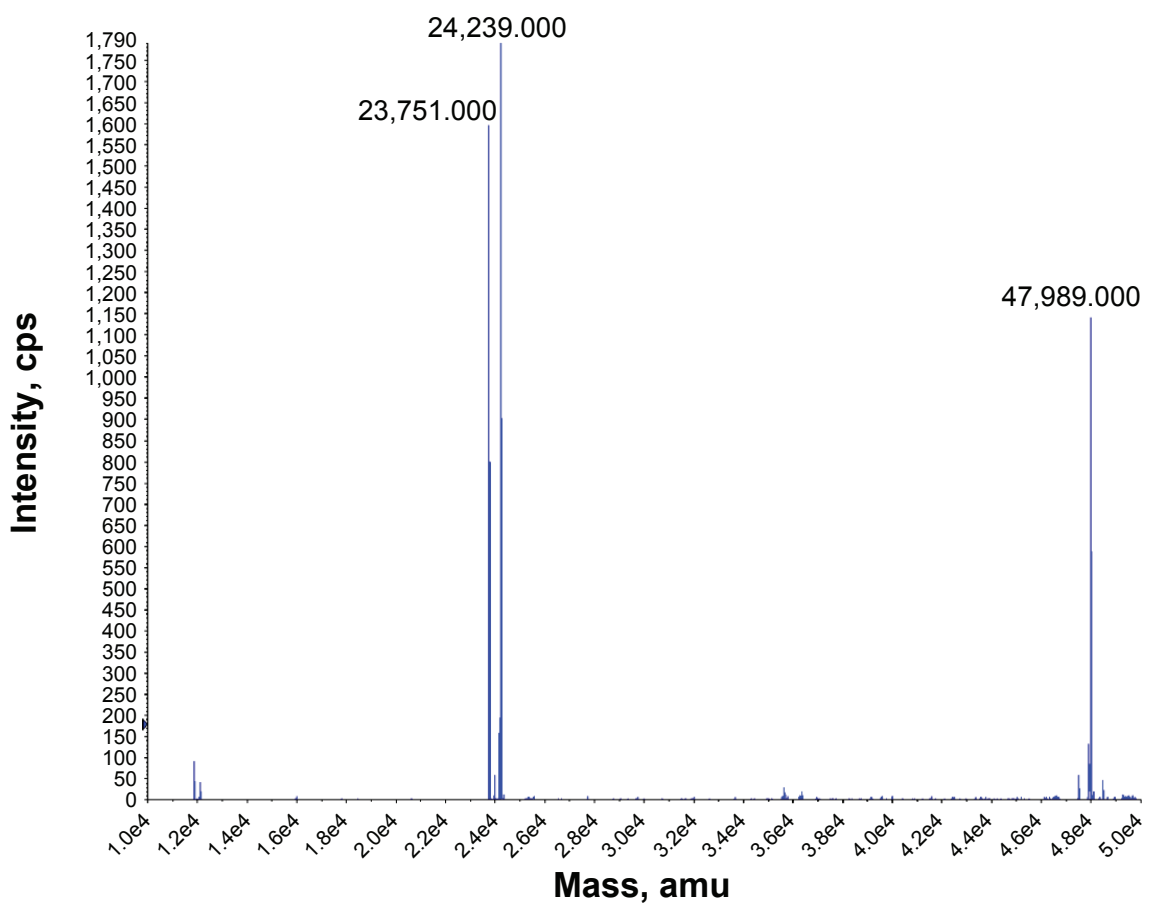

Figure SI (Continued) 
$\mathbf{F}$

Bfab (cysteine)

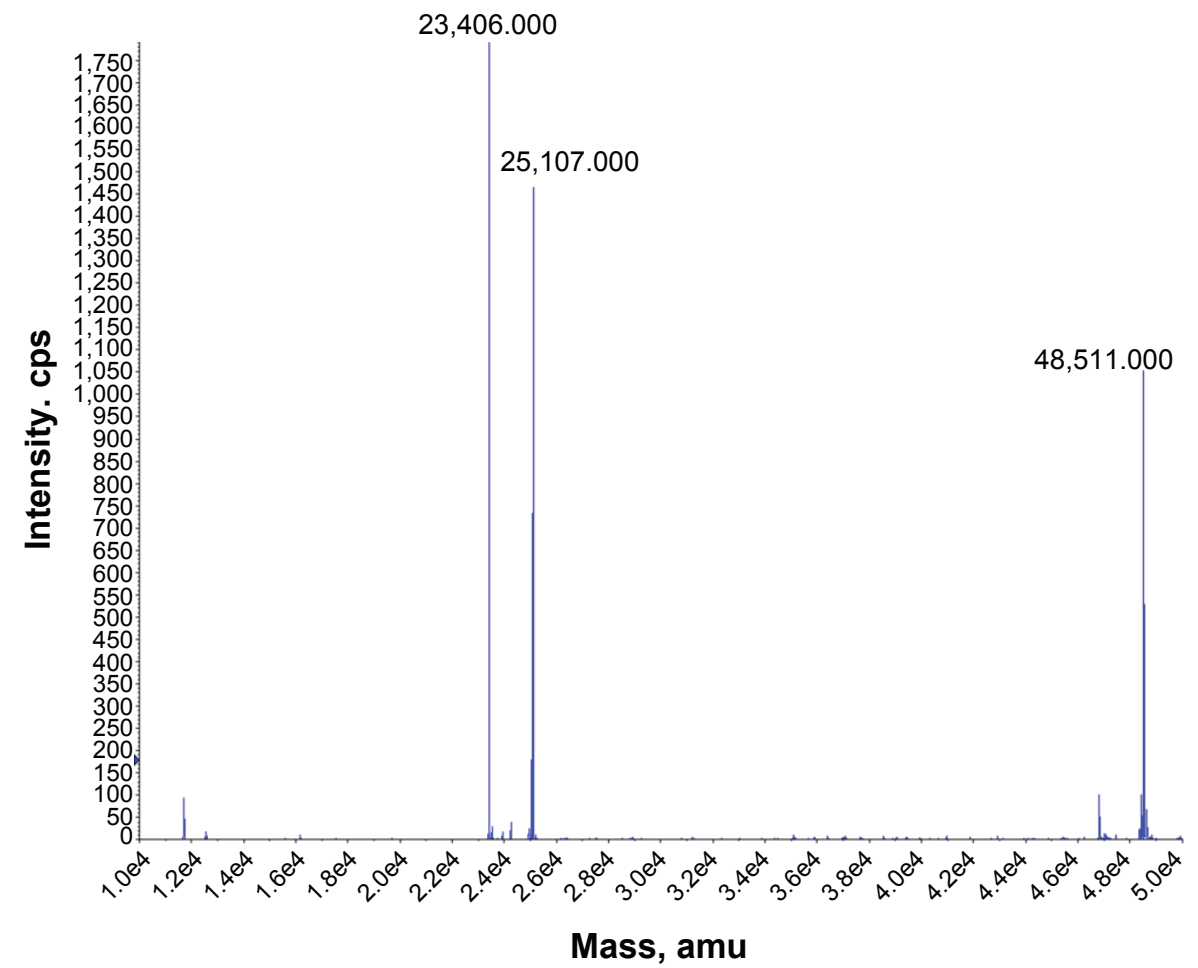

G

Ffab (maleimide-PEG2-biotin)

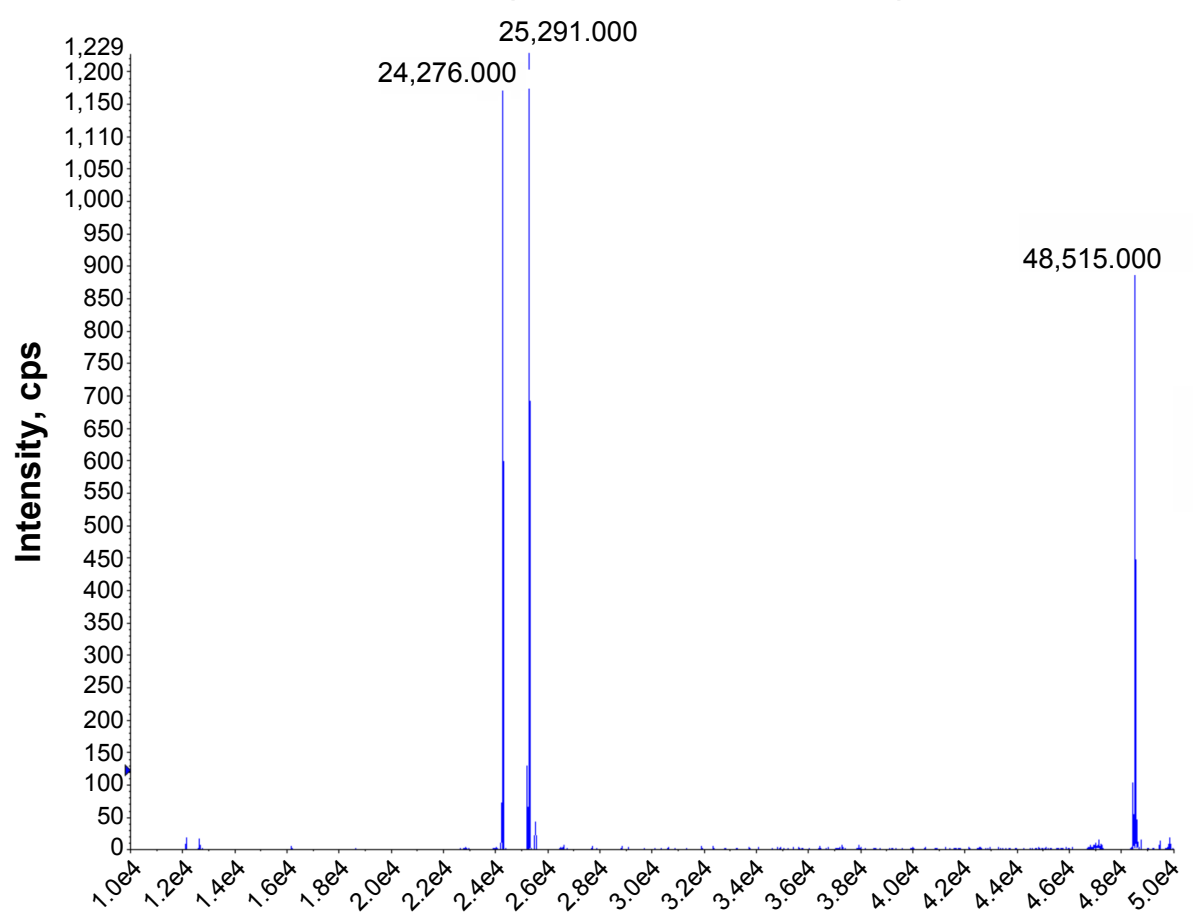

Mass, amu

Figure SI (Continued) 


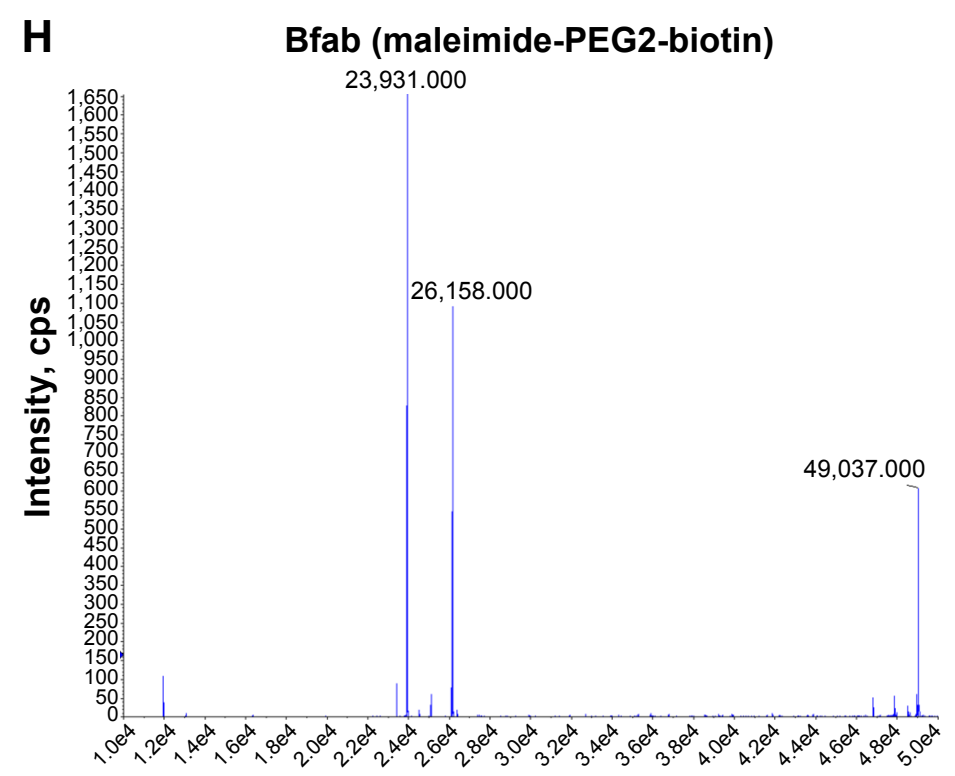

Mass, amu

Figure SI Purity and identity analysis of recombinant fab fragments.

Notes: Size exclusion purification chromatograms of (A) Ffab and (B) Bfab. The blue curves represent UV absorbance at $280 \mathrm{~nm}$, and dashed lines represent the collected monomeric Ffab and Bfab fractions. LC-MS of monomeric SEC fractions from (C) Ffab and (D) Bfab. LC-MS of monomeric (E) Ffab and (F) Bfab following cysteine activation. LC-MS of (G) activated monomeric Ffab and (H) activated monomeric Bfab following conjugation to maleimide-PEG2-biotin. The masses of 24,276 and 23,93। Da correspond to the addition of one maleimide-PEG2-biotin molecule (+526 Da) to the light chains of Ffab and Bfab, respectively. The masses of 25,29I and 26,158 Da correspond to the addition of two maleimide-PEG2-biotin molecules (+I,052 Da) to the heavy chains of Ffab and Bfab, respectively. The masses of 48,5I5 and 49,037 Da correspond to the addition of one maleimide-PEG2-biotin molecule ( $+526 \mathrm{Da}$ ) to intact Ffab and Bfab, respectively.

Abbreviations: fab, an engineered monoclonal antibody fragment; Ffab, Farletuzufab, engineered from monoclonal antibody Farletuzumab; Bfab, Botulifab anti-botulinum toxin fab fragment; UV, ultraviolet; PEG2, polyethylene glycol 2; SEC, size-exclusion chromatography; LC-MS, liquid chromatography-mass spectrometry.

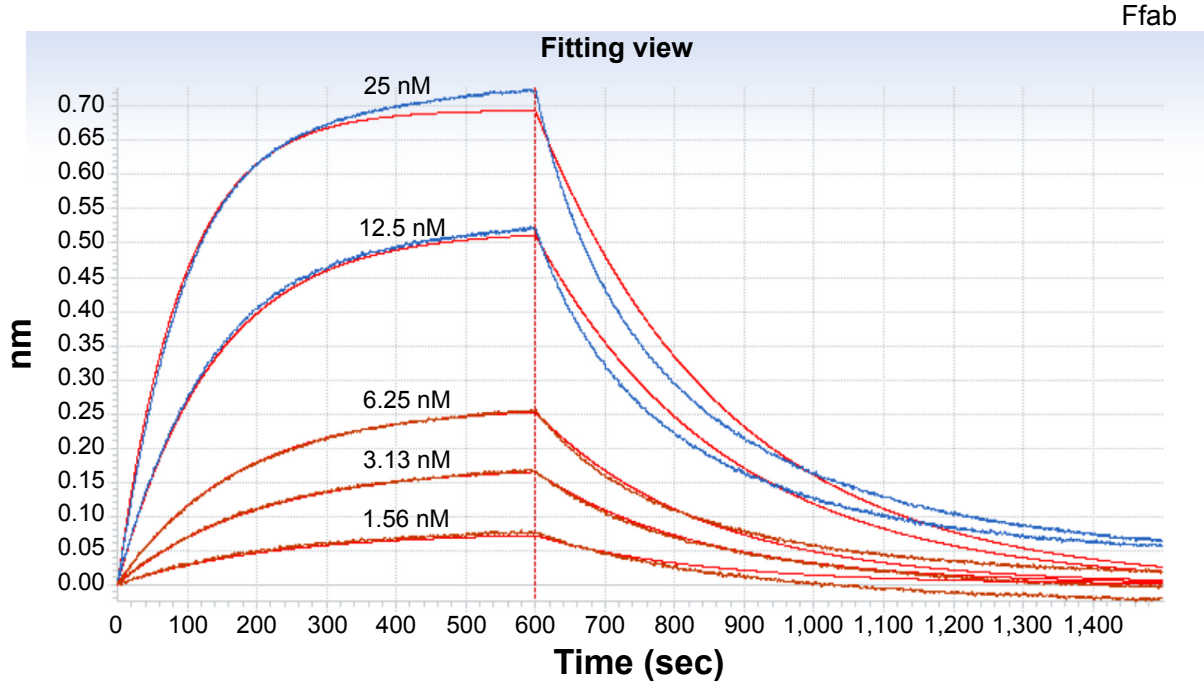

\begin{tabular}{l|l} 
& Ffab \\
\hline $\mathrm{k}_{\text {on }}(1 / \mathrm{M} / \mathrm{s})$ & $2.80 \times 10^{5} \pm 7 \times 10^{3}$ \\
$\mathrm{k}_{\text {off }}(1 / \mathrm{s})$ & $3.91 \times 10^{-3} \pm 2 \times 10^{-5}$ \\
$\mathrm{~K}_{\mathrm{D}}(\mathrm{nM})$ & $14 \pm 0.4$
\end{tabular}

Figure S2 Bio-layer interferometry binding analysis of Ffab antibody fragment.

Notes: Sensorgrams of soluble rFOLR $\alpha$ binding to Ffab immobilized on ForteBio streptavidin biosensor tips. Blue curve indicates measured binding kinetics and red line indicates best-fit curve from kinetic modeling. The best-fit on rate, off rate, and equilibrium dissociation constants are provided below the sensorgrams.

Abbreviations: fab, an engineered monoclonal antibody fragment; Ffab, Farletuzufab, engineered from monoclonal antibody Farletuzumab; Bfab, Botulifab anti-botulinum toxin fab fragment; $r F O L R \alpha$, recombinant folate receptor alpha; sec, seconds. 

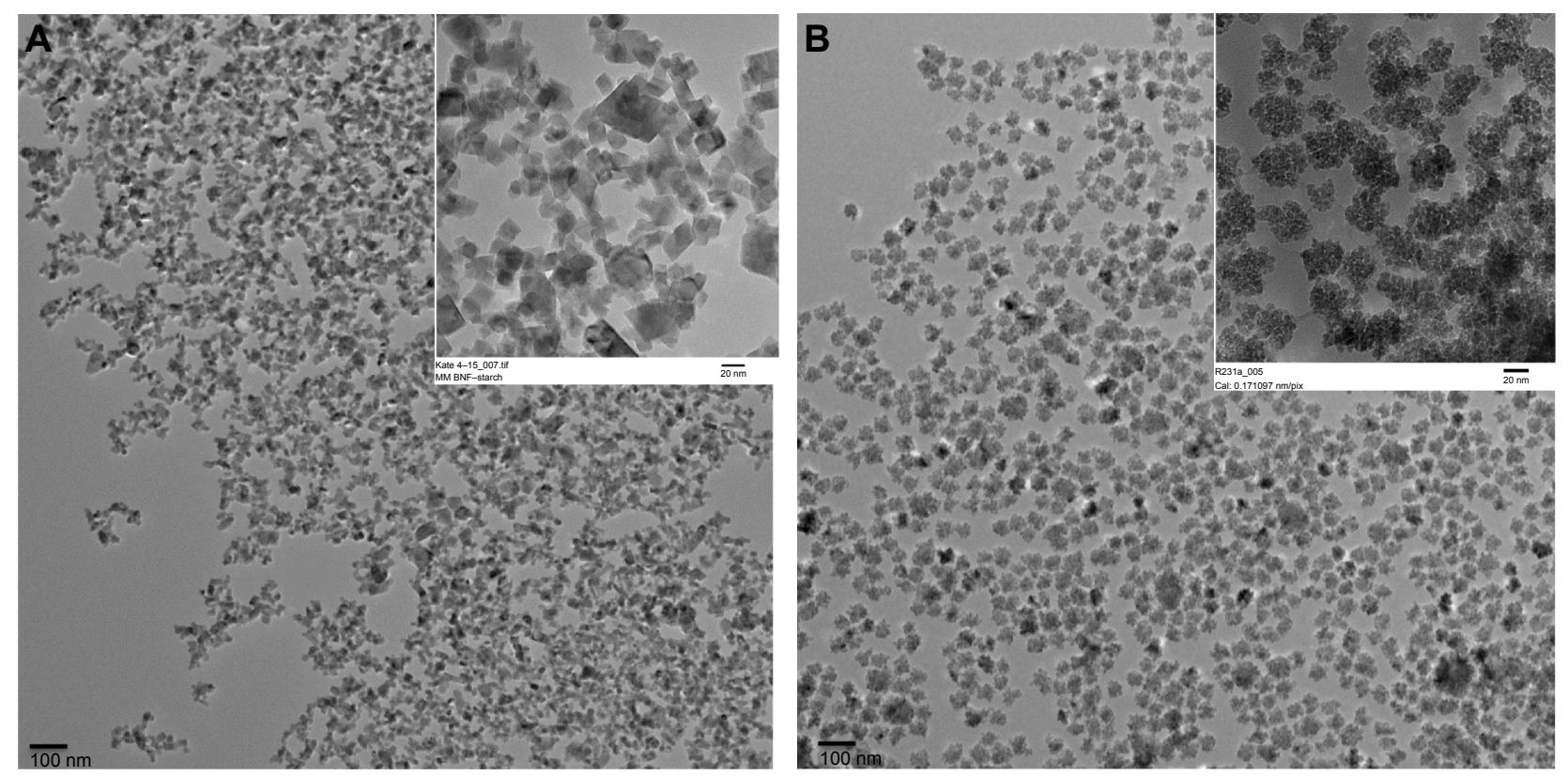

Figure S3 TEM pictures of bulk IONPs.

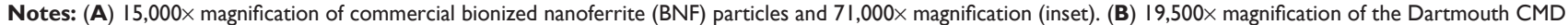
particles and 71,000× magnification (inset).

Abbreviations: TEM, transmission electron microscopy; IONPs, iron oxide nanoparticles; CMD, carboxymethyl-dextran.

A

Size distribution by intensity

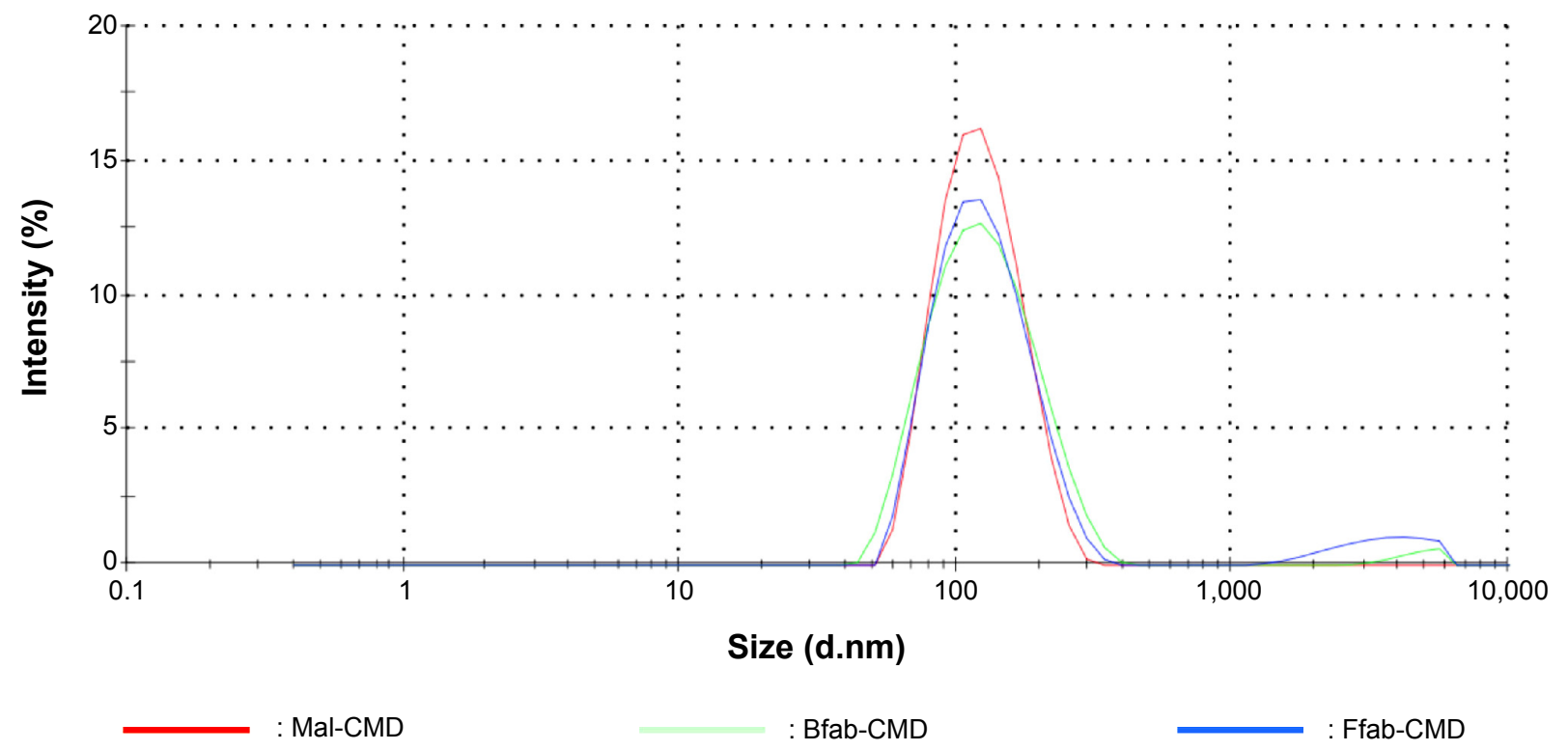

Figure S4 (Continued) 
B

Size distribution by intensity

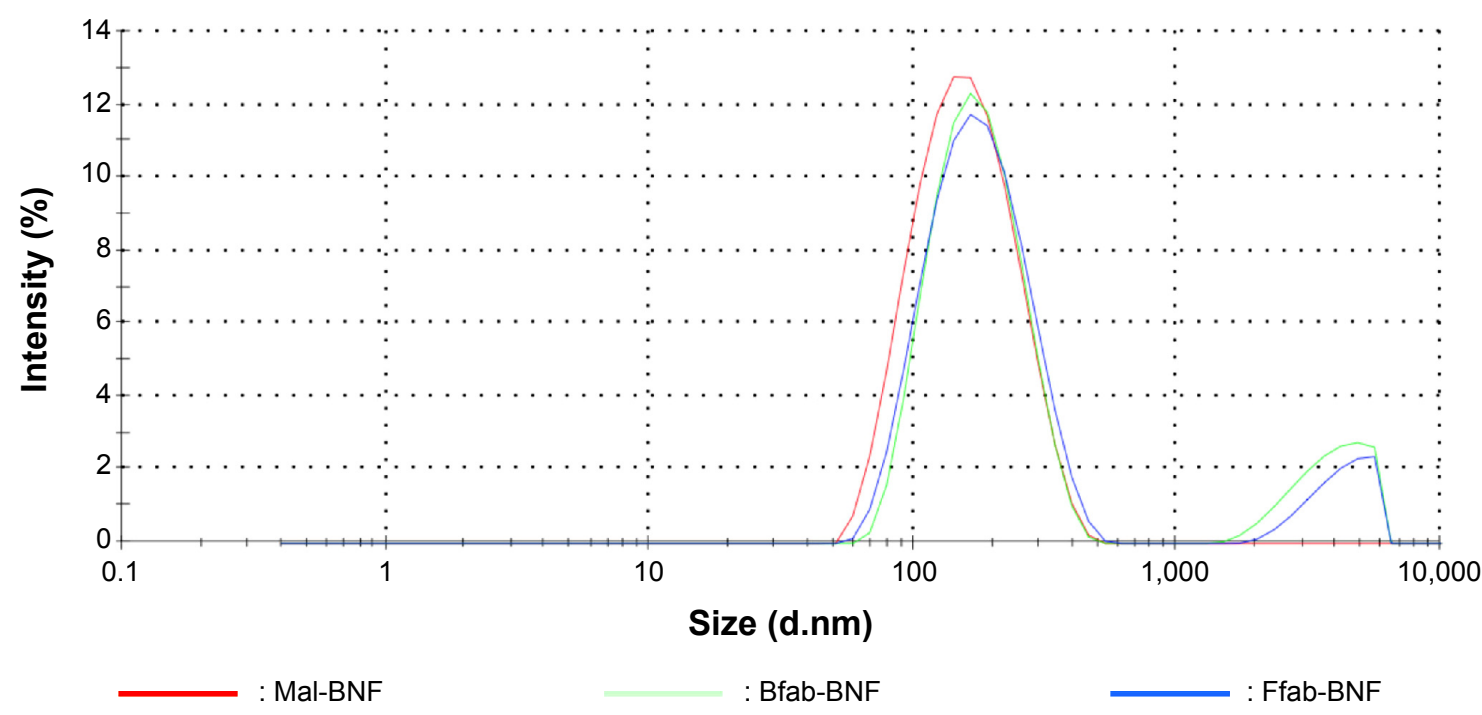

Figure S4 IONP size distribution profile.

Notes: As measured by dynamic light scattering, the intensity size distributions of (A) CMD and (B) BNF IONPs are characterize by mean peaks of II 8 nm and I72 nm, respectively. The red lines are maleimide-conjugated IONPs, the light blue lines are negative control Botulifab-conjugated IONPs, and the dark blue lines are the Farletuzufabconjugated IONPs.

Abbreviations: IONPs, iron oxide nanoparticles; fab, an engineered monoclonal antibody fragment; Ffab, Farletuzufab, engineered from monoclonal antibody Farletuzumab; Bfab, Botulifab anti-botulinum toxin fab fragment; BNF, bionized nanoferrite; CMD, carboxymethyl-dextran; Mal, maleimide.
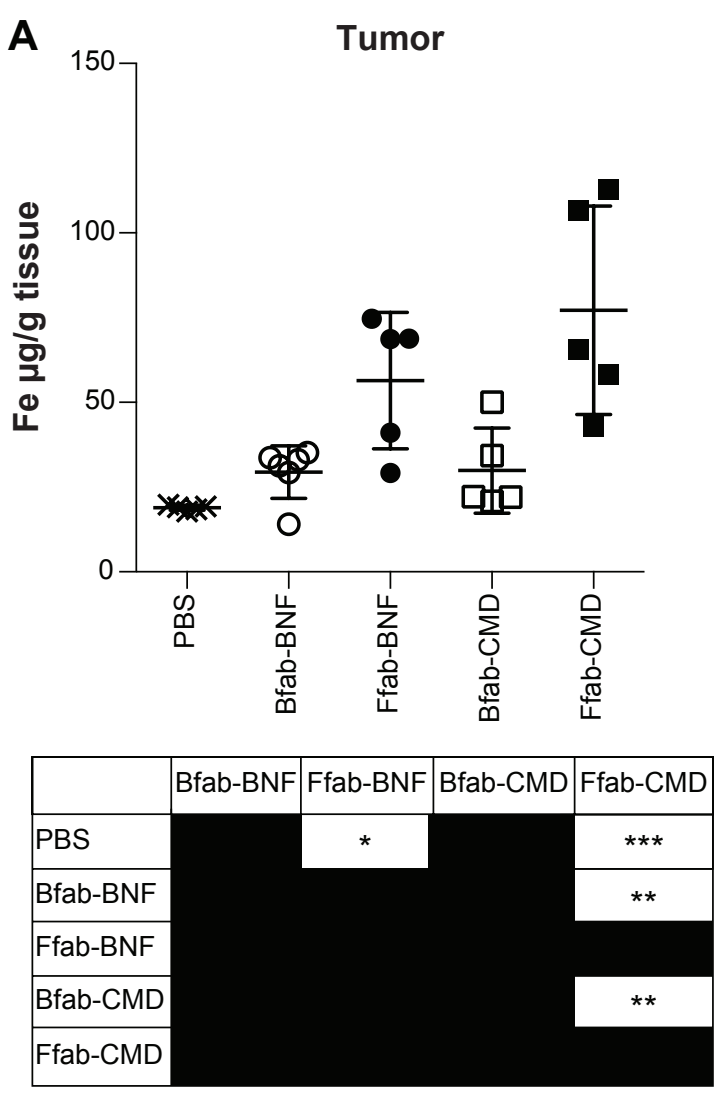

B
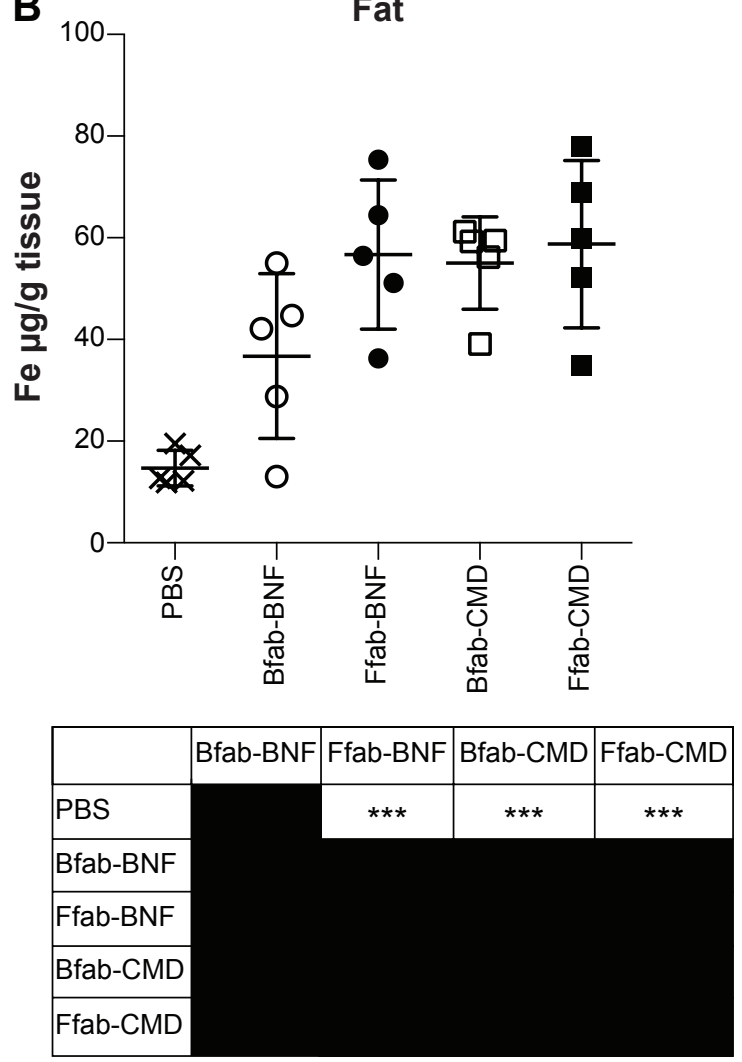

Figure S5 (Continued) 

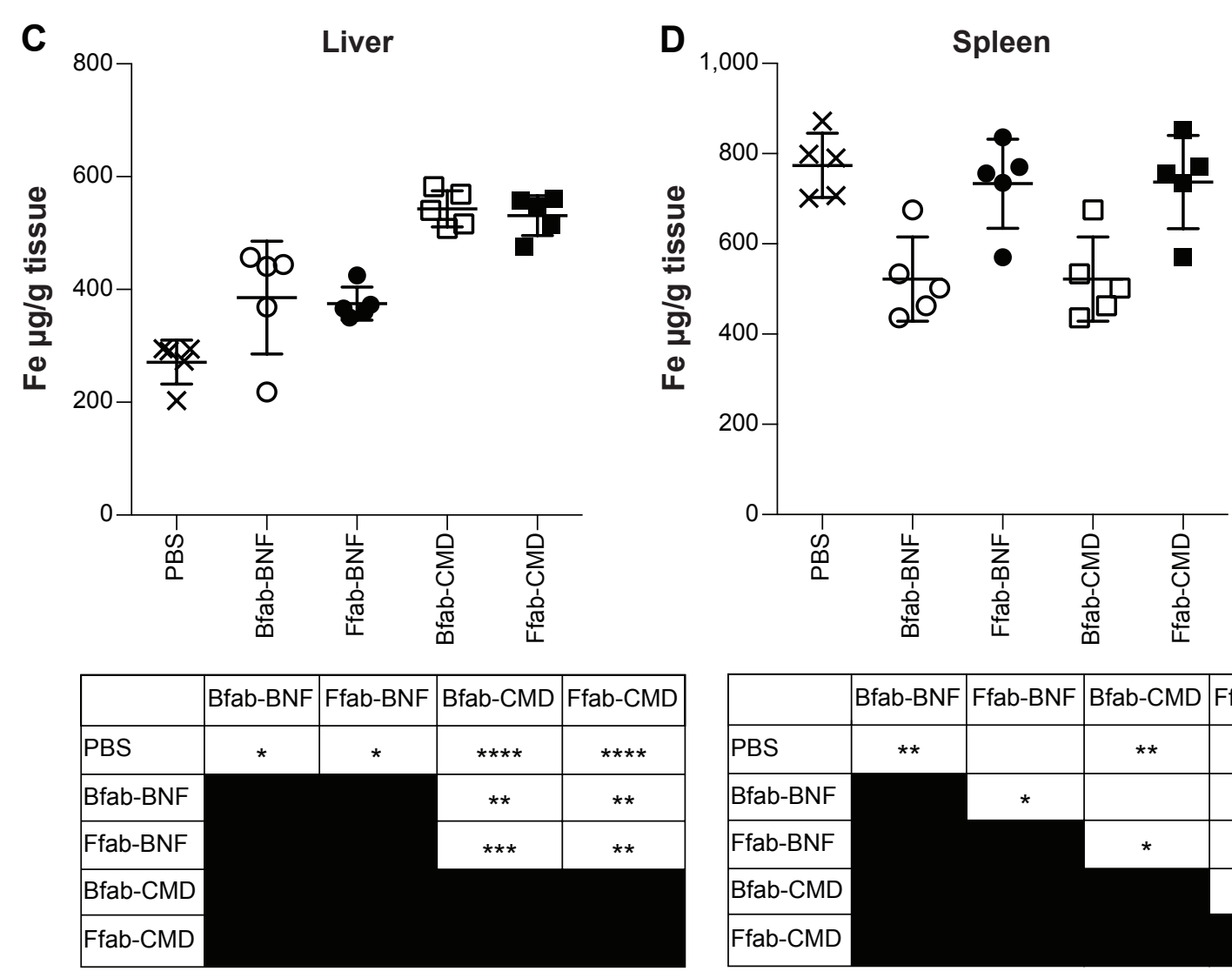

\begin{tabular}{|l|c|c|c|c|}
\hline & Bfab-BNF & Ffab-BNF & Bfab-CMD & Ffab-CMD \\
\hline PBS & $* *$ & & $* *$ & \\
\hline Bfab-BNF & & $*$ & & $*$ \\
\hline Ffab-BNF & & $*$ & \\
\hline Bfab-CMD & & & $*$ \\
\hline Ffab-CMD & & & \\
\hline
\end{tabular}

\section{E}

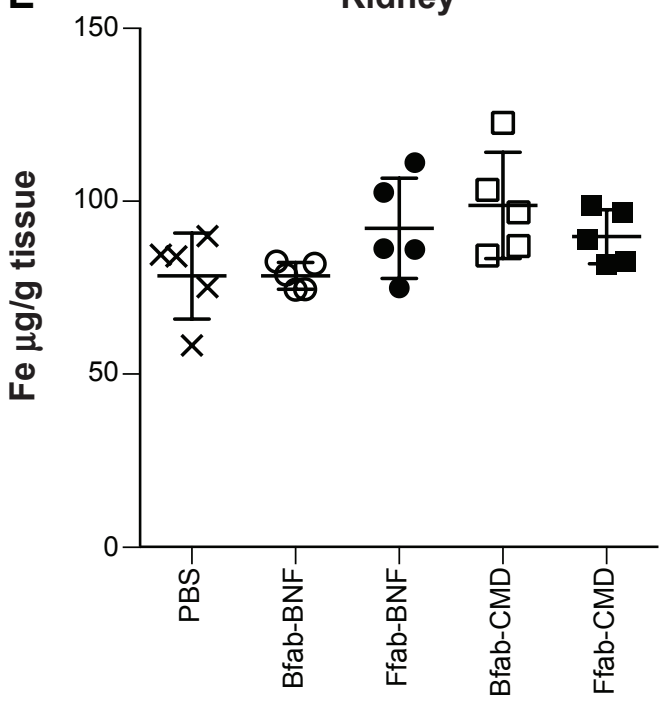

Figure S5 In vivo biodistribution of IONPs.

Notes: Tissue iron concentration (ie, per gram of tissue) is shown for various compartments: (A) tumor; (B) fat; (C) liver; (D) spleen; and (E) kidney. Data obtained by ICP-MS from five mice per group approximately 18 hours post-injection. Statistical significance was analyzed by one-way ANOVA with a Tukey's multiple comparison posttest, and the results of individual comparisons are provided in the tables below each graph. $* * * * P<0.000$ I; $* * * P<0.001 ; * * P<0.0$ I; $* P<0.05$.

Abbreviations: IONPs, iron oxide nanoparticles; ICP-MS, inductively coupled plasma mass spectrometry; ANOVA, analysis of variance; PBS, phosphate-buffered saline; BNF, bionized nanoferrite; CMD, carboxymethyl-dextran; fab, an engineered monoclonal antibody fragment; Ffab, Farletuzufab, engineered from monoclonal antibody Farletuzumab; Bfab, Botulifab anti-botulinum toxin fab fragment. 
International Journal of Nanomedicine

Dovepress

\section{Publish your work in this journal}

The International Journal of Nanomedicine is an international, peerreviewed journal focusing on the application of nanotechnology in diagnostics, therapeutics, and drug delivery systems throughou the biomedical field. This journal is indexed on PubMed Central, MedLine, CAS, SciSearch ${ }^{\circledR}$, Current Contents ${ }^{\circledR} /$ Clinical Medicine,
Journal Citation Reports/Science Edition, EMBase, Scopus and the Elsevier Bibliographic databases. The manuscript management system is completely online and includes a very quick and fair peer-review system, which is all easy to use. Visit http://www.dovepress.com/ testimonials.php to read real quotes from published authors.

Submit your manuscript here: http://www.dovepress.com/international-journal-of-nanomedicine-journal 